Homology, Homotopy and Applications, vol.14(四), 2012, pp.37-61

\title{
MATRIX FACTORIZATIONS OVER PROJECTIVE SCHEMES
}

\author{
JESSE BURKE AND MARK E. WALKER \\ (communicated by Charles A. Weibel)
}

\begin{abstract}
We study matrix factorizations of regular global sections of line bundles on schemes. If the line bundle is very ample relative to a Noetherian affine scheme we show that morphisms in the homotopy category of matrix factorizations may be computed as the hypercohomology of a certain mapping complex. Using this explicit description, we prove an analogue of Orlov's theorem that there is a fully faithful embedding of the homotopy category of matrix factorizations into the singularity category of the corresponding zero subscheme. Moreover, we give a complete description of the image of this functor.
\end{abstract}

\section{Introduction}

Given an element $f$ in a commutative ring $Q$, a matrix factorization of $f$ is a pair of $n \times n$ matrices $(A, B)$ such that $A B=f \cdot I_{n}=B A$. This construction was introduced by Eisenbud in [च] to study modules over the factor ring $R=Q /(f)$. He showed that if $Q$ is a regular local ring and $f$ is nonzero, the minimal free resolution of every finitely generated $R$-module is eventually determined by a matrix factorization [ $\mathbf{2}$, Theorem 6.1]. Buchweitz observed in [⿴囗⿰丿㇄口] (see also [5], 3.9]) that Eisenbud's Theorem implies that there is an equivalence

$$
[M F(Q, f)] \underset{\text { coker }}{\stackrel{\cong}{\cong}} D^{b}(R) / \operatorname{Perf}(R)=: \mathrm{D}_{\mathrm{sg}}(R)
$$

between the homotopy category of matrix factorizations, which is defined analogously to the homotopy category of complexes of modules, and the quotient of the bounded derived category of finitely generated $R$-modules by perfect complexes. Recall that a complex is perfect if it is isomorphic in $D^{b}(R)$ to a bounded complex of finitely generated projective $R$-modules. We call $\mathrm{D}_{\mathrm{sg}}(R)$ the singularity category of $R$, following [5]. The equivalence (四) is induced by sending a matrix factorization $(A, B)$ to the image of the $R$-module coker $A$ in $\mathrm{D}_{\text {sg }}(R)$.

In this paper we study a scheme theoretic generalization of matrix factorizations by replacing $Q$ with a Noetherian separated scheme $X, f$ by a global section $W$ of a line bundle $\mathcal{L}$ on $X$, and $R$ by the zero subscheme $i: Y \hookrightarrow X$ of $W$. A matrix

Received February 2, 2012, revised May 8, 2012; published on October 8, 2012.

2000 Mathematics Subject Classification: 14F05, 13D09, 13 D02.

Key words and phrases: matrix factorization, singularity category.

Article available at http://intlpress.com/HHA/v14/n2/a3 and doi:10.4310/HHA.2012.v14.n2.a3

Copyright (C) 2012, International Press. Permission to copy for private use granted. 
factorization of the triple $(X, \mathcal{L}, W)$ is a pair of locally free sheaves $\mathcal{E}_{1}, \mathcal{E}_{0}$ on $X$ and maps

$$
\mathcal{E}_{1} \stackrel{e_{1}}{\longrightarrow} \mathcal{E}_{0} \stackrel{e_{0}}{\longrightarrow} \mathcal{E}_{1} \otimes \mathcal{L}
$$

such that $e_{0} \circ e_{1}$ and $\left(e_{1} \otimes 1_{\mathcal{L}}\right) \circ e_{0}$ are both multiplication by $W$. The goal of this paper is to explore a generalization of the equivalence $(\mathbb{\square})$ to this scheme-theoretic setting.

The definition of matrix factorizations for schemes given here was introduced in [0]; similar constructions have been studied in $[\mathbf{3}, \mathbf{\Xi}, \mathbf{\nabla}]$. All of these papers have in some way dealt with generalizing $(\mathbb{D})$. Therefore, before we describe our contributions to this question, let us describe what is known. The right hand side of $(\mathbb{\mathbb { C }})$ makes sense for any scheme, in particular the zero subscheme $Y \hookrightarrow X$ of $W$. For the left hand side, one may mimic the affine case and define morphisms analogously to the homotopy category of complexes of sheaves. We write this category as $[M F(X, \mathcal{L}, W)]_{\text {naive }}$, for reasons that will be clear soon. For a matrix factorization $\left(\mathcal{E}_{1} \stackrel{e_{1}}{\longrightarrow} \mathcal{E}_{0} \stackrel{e_{0}}{\longrightarrow} \mathcal{E}_{1} \otimes \mathcal{L}\right)$, multiplication by $W$ on coker $e_{1}$ is zero, and thus we may view coker $e_{1}$ as an object of $\mathrm{D}_{\mathrm{sg}}(Y)$. There is a functor

$$
[M F(X, \mathcal{L}, W)]_{\text {naive }} \rightarrow \mathrm{D}_{\mathrm{sg}}(Y)
$$

that sends a matrix factorization $\left(\mathcal{E}_{1} \stackrel{e_{1}}{\longrightarrow} \mathcal{E}_{0} \stackrel{e_{0}}{\longrightarrow} \mathcal{E}_{1} \otimes \mathcal{L}\right)$ to coker $e_{1}$.

When $X$ is a regular scheme for which every coherent sheaf is the quotient of a locally free sheaf, and $W$ is a regular global section of $\mathcal{L}$ (i.e., $W: \mathcal{O}_{X} \rightarrow \mathcal{L}$ is injective), it is straightforward to see that the functor (四) is essentially surjective. Indeed, as in the affine case, every object of $\mathrm{D}_{\mathrm{sg}}(Y)$ is isomorphic to a coherent sheaf $\mathcal{M}$ that is maximal Cohen-Macaulay (i.e., for each $y \in Y, \mathcal{M}_{y}$ is a maximal Cohen-Macaulay (MCM) module over the ring $\mathcal{O}_{Y, y}$ ), and one may mimic the standard argument in the affine case that associates a matrix factorization to a MCM module: One first takes a surjection $\mathcal{E}_{0} \rightarrow i_{*} \mathcal{M}$ with $\mathcal{E}_{0}$ locally free on $X$. The hypotheses ensure that the kernel $\mathcal{E}_{1}$ will also be locally free. Multiplication by $W$ determines the vertical maps in the diagram

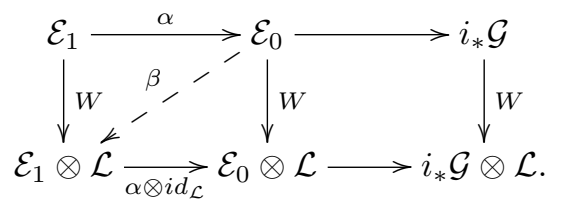

Since the right-most such map is the zero map, there exists a diagonal arrow $\beta$ causing both triangles to commute. We thus obtain a pair of locally free coherent sheaves $\mathcal{E}_{0}, \mathcal{E}_{1}$ on $X$ and morphisms

$$
\mathcal{E}_{1} \stackrel{\alpha}{\longrightarrow} \mathcal{E}_{0} \stackrel{\beta}{\longrightarrow} \mathcal{E}_{1} \otimes \mathcal{L}
$$

such that both compositions $\beta \circ \alpha$ and $\left(\alpha \otimes i d_{\mathcal{L}}\right) \circ \beta$ are multiplication by $W$. This is a matrix factorization of the data $(X, \mathcal{L}, W)$.

In general (『) will not be an equivalence. For observe that if the cokernel of a matrix factorization is locally free, then it is trivial in the singularity category. When $X$ is affine, locally free sheaves are projective and the lifting property of such sheaves allows one to construct a null-homotopy. But in the non-affine case there is no reason 
such a null-homotopy should exist in general, and indeed there are many examples of matrix factorizations that are non-zero in the naive homotopy category but that have a locally free cokernel; see Example एس.

When $X$ is not affine one may, as is done in [ [⿴囗十, $\mathbf{\square}]$, take the Verdier quotient of the naive homotopy category by objects with a locally free cokernel. Let us write this category as $[M F(X, \mathcal{L}, W)]$. Then $[\boldsymbol{\nabla}$, Theorem 3.14], see also [ $\mathbf{\square}$, Theorem 3.5], shows that (『) induces an equivalence

$$
[M F(X, \mathcal{L}, W)] \stackrel{\cong}{\rightarrow} \mathrm{D}_{\mathrm{sg}}(Y) .
$$

In [ $\left[\square\right.$ the line bundle $\mathcal{L}$ is assumed to be $\mathcal{O}_{X}$ but the scheme $X$ is not assumed to be regular. In that case it is shown that the induced functor $[M F(X, \mathcal{L}, W)] \rightarrow \mathrm{D}_{\mathrm{sg}}(Y)$ is fully faithful. See also $[\boldsymbol{\nabla}]$ for a proof of a similar result using exotic derived categories.

A drawback of Verdier quotients is that morphism sets in quotient categories can be difficult to compute. In this paper we offer a different approach to describing the category $[M F(X, \mathcal{L}, W)]$. For every pair of matrix factorizations $\mathbb{E}, \mathbb{F}$ there is mapping complex, denoted by $\underline{\operatorname{Hom}}_{\mathrm{MF}}(\mathbb{E}, \mathbb{F})$, which is a twisted two-periodic complex of locally free sheaves on $X$; see Definition 2.3 for the precise description. We define the category $[M F(X, \mathcal{L}, W)]_{\mathbb{H}}$ to have objects all matrix factorizations and for two such objects $\mathbb{E}, \mathbb{F}$, morphisms between them are given by

$$
\operatorname{Hom}_{[M F]_{\mathbb{H}}}(\mathbb{E}, \mathbb{F})=\mathbb{H}^{0} \underline{\operatorname{Hom}}_{\mathrm{MF}}(\mathbb{E}, \mathbb{F}),
$$

where $\mathbb{H}^{0}$ denotes hypercohomology in degree 0 . There is a composition which is associative and unital.

Recall that a scheme $X$ is projective over a ring $Q$ if there is a closed embedding $j: X \hookrightarrow \mathbb{P}_{Q}^{m}$ for some $m \geqslant 0$. In this case, we say that $\mathcal{O}_{X}(1):=j^{*} \mathcal{O}_{\mathbb{P}_{Q}^{m}}(1)$ is the corresponding very ample line bundle. Our first main result shows that in this case the two homotopy categories coincide.

Theorem 1. Let $X$ be a scheme that is projective over a Noetherian ring and $\mathcal{L}=$ $\mathcal{O}_{X}(1)$ the corresponding very ample line bundle. For a global section $W$ of $\mathcal{L}$ there is an equivalence of categories

$$
[M F(X, \mathcal{L}, W)] \stackrel{\cong}{\rightrightarrows}[M F(X, \mathcal{L}, W)]_{\mathbb{H}} .
$$

Using this concrete description of the morphisms in the homotopy category, we are able to give another proof of (an analogue of) [⿴囗十 Theorem 3.4] (which assumed that $\left.\mathcal{L}=\mathcal{O}_{X}\right)$ when $X$ is projective over a Noetherian ring and $\mathcal{L}=\mathcal{O}_{X}(1)$.

Theorem 2. Let $X$ be a scheme that is projective over a Noetherian ring of finite Krull dimension, $\mathcal{L}=\mathcal{O}_{X}(1)$ the corresponding very ample line bundle, and $W$ a regular global section of $\mathcal{L}$. Define $i: Y \hookrightarrow X$ to be zero subscheme of $W$. There is a functor

$$
\overline{\text { coker }: ~}[M F(X, \mathcal{L}, W)] \rightarrow \mathrm{D}_{\mathrm{sg}}(Y),
$$

which sends a matrix factorization $\left(\mathcal{E}_{1} \stackrel{e_{1}}{\longrightarrow} \mathcal{E}_{0} \stackrel{e_{0}}{\longrightarrow} \mathcal{E}_{1} \otimes \mathcal{L}\right)$ to coker $e_{1}$, and which is fully faithful. The essential image is given by the objects $\mathcal{C}$ in $\mathrm{D}_{\mathrm{sg}}(Y)$ such that $i_{*} \mathcal{C}$ is perfect on $X$. In particular, if $X$ is regular then $\overline{\text { coker }}$ is an equivalence. 
In [四] Orlov remarks that it would be interesting to understand the difference between $[M F(X, \mathcal{L}, W)]$ and $\mathrm{D}_{\mathrm{sg}}(Y)$. The above theorem shows that, when $X$ is projective over an affine scheme and $\mathcal{L}=\mathcal{O}_{X}(1)$, the difference is exactly the class of objects in $\mathrm{D}_{\mathrm{sg}}(Y)$ that are not perfect over $X$.

We came to these results studying affine complete intersection rings. Let $Q$ be a regular ring, $f_{1}, \ldots, f_{c}$ a regular sequence of elements in $Q$, and set $R=Q /\left(f_{1}, \ldots, f_{c}\right)$. Define $X=\mathbb{P}_{Q}^{c-1}=\operatorname{Proj} Q\left[T_{1}, \ldots, T_{c}\right], \mathcal{L}=\mathcal{O}_{X}(1), W=\sum_{i} f_{i} T_{i} \in \Gamma(X, \mathcal{L})$ and $Y \hookrightarrow$ $X$ the zero subscheme of $W$. Orlov showed in [6, Theorem 2.1] that there is an equivalence

$$
\mathrm{D}_{\mathrm{sg}}(R) \stackrel{\cong}{\longrightarrow} \mathrm{D}_{\mathrm{sg}}(Y)
$$

Composing this with the equivalence of Theorem 2 we obtain an equivalence

$$
\mathrm{D}_{\mathrm{sg}}(R) \stackrel{\cong}{\longrightarrow}[M F(X, \mathcal{L}, W)]
$$

In a companion paper to this one we will use this equivalence and the explicit description of the Hom-sets in $[M F(X, \mathcal{L}, W)]$ given here to study the cohomology of modules over $R$.

\section{Acknowledgements}

The authors thank Greg Stevenson for carefully reading a preliminary version of this paper and offering helpful comments on it.

\section{The generalized category of matrix factorizations}

Throughout $X$ will denote a Noetherian separated scheme and $\mathcal{L}$ a line bundle on $X$. To simplify notation, even if $\mathcal{L}$ is not very ample, for a quasi-coherent sheaf $\mathcal{G}$ (or a complex of such) on $X$ and integer $n$, we will write $\mathcal{G}(n)$ for $\mathcal{G} \otimes \mathcal{O}_{X} \mathcal{L}^{\otimes n}$. (Recall $\mathcal{L}^{\otimes-n}:=\underline{\operatorname{Hom}}_{\mathcal{O}_{X}}\left(\mathcal{L}^{\otimes n}, \mathcal{O}_{X}\right)$ for $n \geqslant 1$.) In particular, $\mathcal{O}(1)=\mathcal{L}$. Similarly, if $f$ is morphism of (complexes of) quasi-coherent sheaves, then $f(1)=f \otimes i d_{\mathcal{L}}$.

The following definition first appeared in [ $\mathbf{7}]$.

Definition 2.1. Let $W$ be a global section of $\mathcal{L}$. A matrix factorization $\mathbb{E}=\left(\mathcal{E}_{1} \stackrel{e_{1}}{\longrightarrow}\right.$ $\left.\mathcal{E}_{0} \stackrel{e_{0}}{\rightarrow} \mathcal{E}_{1}(1)\right)$ of the triple $(X, \mathcal{L}, W)$ consists of a pair of locally free coherent sheaves $\mathcal{E}_{1}, \mathcal{E}_{0}$ on $X$ and morphisms $e_{1}: \mathcal{E}_{1} \rightarrow \mathcal{E}_{0}$ and $e_{0}: \mathcal{E}_{0} \rightarrow \mathcal{E}_{1}(1)$ such that $e_{0} \circ e_{1}$ and $e_{1}(1) \circ e_{0}$ are multiplication by $W$. A strict morphism of matrix factorizations from $\left(\mathcal{E}_{1} \rightarrow \mathcal{E}_{0} \rightarrow \mathcal{E}_{1}(1)\right)$ to $\left(\mathcal{F}_{1} \rightarrow \mathcal{F}_{0} \rightarrow \mathcal{F}_{1}(1)\right)$ is a pair of maps $\mathcal{E}_{0} \rightarrow \mathcal{F}_{0}, \mathcal{E}_{1} \rightarrow \mathcal{F}_{1}$ causing the evident pair of squares to commute. Matrix factorizations and strict morphisms of such form a category which we write $M F(X, \mathcal{L}, W)_{\text {exact }}$ or just $M F_{\text {exact }}$ for short.

The larger category, with objects matrix factorizations of arbitrary coherent sheaves and arrows strict morphisms defined in the same way as above, is an abelian category. The category $M F_{\text {exact }}$ is a full subcategory of this abelian category and is closed under extensions, and hence $M F_{\text {exact }}$ has the structure of an exact category in the sense of Quillen [ $[\mathbf{0}]$. A sequence $0 \rightarrow \mathbb{E}^{\prime} \rightarrow \mathbb{E} \rightarrow \mathbb{E}^{\prime \prime} \rightarrow 0$ in $M F_{\text {exact }}$ is a short exact sequence if it determines a short exact sequence of locally free coherent sheaves in both degrees. 
Definition 2.2. A twisted periodic complex of locally free coherent sheaves for $(X, \mathcal{L})$ is a chain complex $\mathcal{C}$ of locally free coherent sheaves on $X$ together with a specified isomorphism $\alpha: \mathcal{C}[2] \stackrel{\cong}{\rightarrow} \mathcal{C}(1)$, where we use the convention that $\mathcal{C}[2]^{i}=\mathcal{C}^{i+2}$. The category $\operatorname{TPC}(X, \mathcal{L})$ has as objects twisted periodic complexes and a morphism of such objects is a chain map that commutes with the isomorphisms in the evident sense. There is an equivalence

$$
\operatorname{TPC}(X, \mathcal{L}) \cong M F(X, \mathcal{L}, 0)_{\text {exact }}
$$

given by sending $(\mathcal{C}, \alpha)$ to $\mathcal{C}^{-1} \stackrel{d}{\rightarrow} \mathcal{C}^{0} \stackrel{\alpha^{-1} \circ d}{\longrightarrow} \mathcal{C}^{-1}(1)$.

The most important example of a twisted periodic complex, for us, is the following:

Definition 2.3. Let $\mathbb{E}=\left(\mathcal{E}_{1} \stackrel{e_{1}}{\longrightarrow} \mathcal{E}_{0} \stackrel{e_{0}}{\longrightarrow} \mathcal{E}_{1}(1)\right)$ and $\mathbb{F}=\left(\mathcal{F}_{1} \stackrel{f_{1}}{\longrightarrow} \mathcal{F}_{0} \stackrel{f_{0}}{\longrightarrow} \mathcal{F}_{1}(1)\right)$ be matrix factorizations for $(X, \mathcal{L}, W)$. We define the mapping complex of $\mathbb{E}, \mathbb{F}$, written $\underline{\operatorname{Hom}}_{\mathrm{MF}}(\mathbb{E}, \mathbb{F})$, to be the following twisted periodic complex of locally free sheaves:

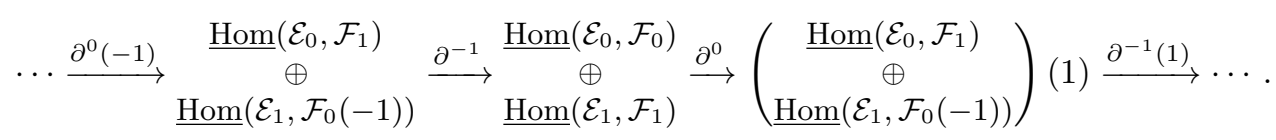

Here, Hom denotes the sheaf of homomorphisms between two coherent sheaves on $X$ and $\underline{\operatorname{Hom}}\left(\mathcal{E}_{0}, \mathcal{F}_{0}\right) \oplus \underline{\operatorname{Hom}}\left(\mathcal{E}_{1}, \mathcal{F}_{1}\right)$ lies in degree 0 . The differentials are given by

$$
\partial^{-1}=\left[\begin{array}{cc}
\left(f_{1}\right)_{*} & -e_{0}^{*} \\
-e_{1}^{*} & \left(f_{0}\right)_{*}
\end{array}\right] \quad \text { and } \quad \partial^{0}=\left[\begin{array}{cc}
\left(f_{0}\right)_{*} & e_{0}^{*} \\
e_{1}^{*} & \left(f_{1}\right)_{*}
\end{array}\right] \text {, }
$$

using the canonical isomorphisms

$$
\underline{\operatorname{Hom}}\left(\mathcal{E}_{i}, \mathcal{F}_{j}(1)\right) \cong \underline{\operatorname{Hom}}\left(\mathcal{E}_{i}, \mathcal{F}_{j}\right)(1) \cong \underline{\operatorname{Hom}}\left(\mathcal{E}_{i}(-1), \mathcal{F}_{j}\right)
$$

and

$$
\left(\begin{array}{c}
\underline{\operatorname{Hom}}\left(\mathcal{E}_{0}, \mathcal{F}_{1}\right) \\
\oplus \\
\underline{\operatorname{Hom}}\left(\mathcal{E}_{1}, \mathcal{F}_{0}(-1)\right)
\end{array}\right)(1) \cong \begin{gathered}
\underline{\operatorname{Hom}}\left(\mathcal{E}_{0}, \mathcal{F}_{1}(1)\right) \\
\oplus \\
\underline{\operatorname{Hom}}\left(\mathcal{E}_{1}, \mathcal{F}_{0}\right)
\end{gathered}
$$

One checks that $\partial^{0} \circ \partial^{-1}$ and $\partial^{-1}(1) \circ \partial^{0}$ are both 0 , and hence $\underline{\operatorname{Hom}}_{\mathrm{MF}}(\mathbb{E}, \mathbb{F})$ is in fact a twisted periodic complex.

Note that there is an isomorphism

$$
\operatorname{Hom}_{M F_{\text {exact }}}(\mathbb{E}, \mathbb{F}) \cong Z^{0}\left(\Gamma\left(X, \underline{\operatorname{Hom}}_{\mathrm{MF}}(\mathbb{E}, \mathbb{F})\right)\right)
$$

where $\Gamma\left(X, \underline{\operatorname{Hom}}_{\mathrm{MF}}(\mathbb{E}, \mathbb{F})\right)$ is the complex of abelian groups obtained by applying the global sections functor degree-wise to $\underline{\operatorname{Hom}}_{\mathrm{MF}}(\mathbb{E}, \mathbb{F})$, and $Z^{0}$ denotes the cycles in degree 0 .

Definition 2.4. A strict morphism $\left(g_{1}, g_{0}\right): \mathbb{E} \rightarrow \mathbb{F}$ is nullhomotopic if there are maps 
$s: \mathcal{E}_{0} \rightarrow \mathcal{F}_{1}$ and $t: \mathcal{E}_{1}(1) \rightarrow \mathcal{F}_{0}$ as in the diagram below

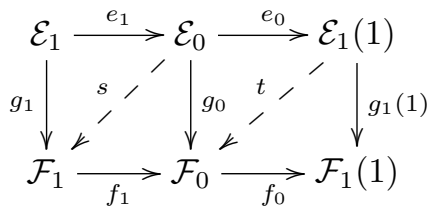

such that

$$
g_{1}=s \circ e_{1}+f_{0}(-1) \circ t(-1) \text { and } g_{0}=f_{1} \circ s+t \circ e_{0} .
$$

Two strict morphisms are homotopic if their difference is nullhomotopic.

The naive homotopy category of matrix factorizations, written

$$
[M F(X, \mathcal{L}, W)]_{\text {naive }},
$$

is the category with the same objects as $M F(X, \mathcal{L}, W)_{\text {exact }}$ and arrows given by strict morphisms modulo homotopy. Equivalently, for objects $\mathbb{E}, \mathbb{F}$ :

$$
\operatorname{Hom}_{[M F]_{\text {naive }}}(\mathbb{E}, \mathbb{F})=H^{0} \Gamma\left(X, \underline{\operatorname{Hom}}_{\mathrm{MF}}(\mathbb{E}, \mathbb{F})\right) .
$$

Definition 2.5. The shift functor on $[M F(X, \mathcal{L}, W)]_{\text {naive, }}$ written [1], is the endofunctor given on objects by

$$
\left(\mathcal{E}_{1} \stackrel{e_{1}}{\longrightarrow} \mathcal{E}_{0} \stackrel{e_{0}}{\longrightarrow} \mathcal{E}_{1}(1)\right)[1]=\left(\mathcal{E}_{0} \stackrel{-e_{0}}{\longrightarrow} \mathcal{E}_{1}(1) \stackrel{-e_{1}(1)}{\longrightarrow} \mathcal{E}_{0}(1)\right) .
$$

The cone of a strict morphism $f=\left(g_{1}, g_{0}\right): \mathbb{E} \rightarrow \mathbb{F}$ is the matrix factorization

$$
\text { cone }(f)=\left(\mathcal{E}_{0} \oplus \mathcal{F}_{1} \stackrel{\left[\begin{array}{cc}
-e_{0} & 0 \\
g_{0} & f_{1}
\end{array}\right]}{\longrightarrow} \mathcal{E}_{1}(1) \oplus \mathcal{F}_{0} \stackrel{\left[\begin{array}{cc}
-e_{1}(1) & 0 \\
g_{1}(1) & f_{0}
\end{array}\right]}{\longrightarrow} \mathcal{E}_{0}(1) \oplus \mathcal{F}_{1}(1)\right) .
$$

There are maps $\mathbb{F} \rightarrow \operatorname{cone}(f) \rightarrow \mathbb{E}[1]$ defined in the usual manner, and we define a distinguished triangle to be a triangle in $[M F]_{\text {naive }}$ isomorphic to one of the form

$$
\mathbb{E} \stackrel{f}{\rightarrow} \mathbb{F} \rightarrow \operatorname{cone}(f) \rightarrow \mathbb{E}[1] .
$$

As remarked in [ $\left[\mathbf{7}\right.$, these structures make $[M F(X, \mathcal{L}, W)]_{\text {naive }}$ into a triangulated category, a fact one can check directly by mimicking the proof for the homotopy category of complexes in an abelian category.

Example 2.6. Let $Q$ be a Noetherian $\operatorname{ring}, X=\operatorname{Spec} Q, \mathcal{L}=\mathcal{O}_{X}$, and $W$ an element of $Q$. Then a matrix factorization is a pair of projective $Q$-modules $E_{1}, E_{0}$ and maps

$$
E_{1} \longleftrightarrow E_{0}
$$

such that composition in either direction is multiplication by $W$. The category $[M F(X, \mathcal{L}, W)]_{\text {naive }}$ is the homotopy category of matrix factorizations, as defined for instance in [5, 3.1], where it is denoted $\operatorname{HMF}(Q, W)$.

For a point $x \in X$, we may localize an object $\mathbb{E}$ of $M F(X, \mathcal{L}, W)_{\text {exact }}$ at $x$ in the evident manner to obtain an object of $\operatorname{MF}\left(\mathcal{O}_{X, x}, \mathcal{L}_{x}, W_{x}\right)_{\text {exact }}$. It is clear that the 
functor

$$
M F(X, \mathcal{L}, W)_{\text {exact }} \rightarrow M F\left(\mathcal{O}_{X, x}, \mathcal{L}_{x}, W_{x}\right)_{\text {exact }}, \mathbb{E} \mapsto \mathbb{E}_{x}
$$

preserves homotopies, and in this way we obtain a triangulated functor

$$
[M F(X, \mathcal{L}, W)]_{\text {naive }} \rightarrow\left[M F\left(\mathcal{O}_{X, x}, \mathcal{L}_{x}, W_{x}\right)\right]_{\text {naive }} .
$$

Definition 2.7. A map of matrix factorizations $\mathbb{E} \rightarrow \mathbb{E}^{\prime}$ in $[M F(X, \mathcal{L}, W)]_{\text {naive }}$ is a weak equivalence if for each $x \in X$, the map $\mathbb{E}_{x} \rightarrow \mathbb{E}_{x}^{\prime}$ is an isomorphism in the category $\left[M F\left(\mathcal{O}_{X, x}, \mathcal{L}_{x}, W_{x}\right)\right]_{\text {naive }}$.

A matrix factorization $\mathbb{F}$ is locally contractible if the unique map $0 \rightarrow \mathbb{F}$ is a weak equivalence. This is equivalent to the condition that $\mathbb{F}_{x}$ is contractible for all $x \in X$.

Remark 2.8. It is asserted in [3], 2.6] that there is a model category structure for the evident generalization of the notion of matrix factorizations for $(X, \mathcal{L}, W)$ in which the objects involve a pair of arbitrary quasi-coherent sheaves. This larger category, which is closed under all small coproducts, plays an important role in the work of Positselski [8] and Lin and Pomerleano [3].

For a global section $W$ of a line bundle $\mathcal{L}$, the zero subscheme of $W$ is the subscheme of $X$ determined by the ideal sheaf given as the image of $W^{\vee}: \mathcal{L}^{\vee} \rightarrow \mathcal{O}_{X}$.

Proposition 2.9. Assume $X$ is a Noetherian scheme, $\mathcal{L}$ is a line bundle on $X$, and $W$ is a regular global section of $\mathcal{L}$, i.e., the map $W: \mathcal{O}_{X} \rightarrow \mathcal{L}$ is injective. Let $i: Y \hookrightarrow X$ be the zero subscheme of $W$. Consider the following conditions on a matrix factorization $\mathbb{E}=\left(\mathcal{E}_{1} \stackrel{e_{1}}{\longrightarrow} \mathcal{E}_{0} \stackrel{e_{0}}{\longrightarrow} \mathcal{E}_{1}(1)\right)$ :

(1) $\mathbb{E} \cong 0$ in the category $[M F(X, \mathcal{L}, W)]_{\text {naive }}$.

(2) The canonical surjection $p: i^{*} \mathcal{E}_{0} \rightarrow i^{*} \operatorname{coker}\left(e_{1}\right)$ of coherent $\mathcal{O}_{Y}$-sheaves splits.

(3) $\mathbb{E}$ is locally contractible.

(4) $i^{*} \operatorname{coker}\left(e_{1}\right)$ is a locally free coherent sheaf on $Y$.

In general, we have $1 \Rightarrow 2 \Rightarrow 3 \Longleftrightarrow 4$. If $X$ is affine then all four conditions are equivalent.

Remark 2.10. Note that multiplication by $W$ annihilates coker $\left(e_{1}\right)$, and hence

$$
i_{*} i^{*} \operatorname{coker}\left(e_{1}\right) \cong \operatorname{coker}\left(e_{1}\right)
$$

via the canonical map.

Proof. We first observe that the proof of [5, 3.8] applies to show that (4) implies (1) when $X$ is affine.

We next prove that (1) implies (2). Let $\mathcal{M}=i^{*} \operatorname{coker}\left(e_{1}\right)=\operatorname{coker}\left(i^{*} e_{1}\right)$. If $\mathbb{E} \cong 0$, then $\mathbb{E}$ has a contracting homotopy, given by $s: \mathcal{E}_{0} \rightarrow \mathcal{E}_{1}$ and $t: \mathcal{E}_{1}(1) \rightarrow \mathcal{E}_{0}$ satisfying $e_{1} s+t e_{0}=i d$ and $s(1) e_{1}(1)+e_{0} t=i d$. As in the argument in the proof of [5, 3.7], since $i^{*}\left(t e_{0} e_{1}\right)=0$ and $\mathcal{M}$ is the cokernel of $i^{*}\left(e_{1}\right)$, there is a map $j: \mathcal{M} \rightarrow i^{*} \mathcal{E}_{0}$ such that $j p=i^{*}\left(t e_{0}\right)$. But then

$$
p j p=p i^{*}\left(t e_{0}\right)=p i^{*}\left(i d-e_{1} s\right)=p
$$

since $p i^{*}\left(e_{1}\right)=0$. Since $p$ is onto, we have $p j=i d_{\mathcal{M}}$. 
If $\left(i^{*} \text { coker } \mathbb{E}\right)_{x}$ is a free $\mathcal{O}_{Y, x}$ module for all $x \in Y$, then, since (4) implies (1) in the affine case, we conclude that $\mathbb{E}_{x}=0 \in\left[M F\left(\mathcal{O}_{X, x}, \mathcal{L}_{X}, W_{x}\right)\right]$. This proves that (4) implies 3. It is clear that (2) implies (4) and hence that (2) implies (3).

Finally, if $\mathbb{E}_{x} \cong 0$, then since (1) implies (2), we see that the map $p_{x}:\left(i^{*} \mathcal{E}_{0}\right)_{x} \rightarrow$ $\left(i^{*} \operatorname{coker}\left(e_{1}\right)\right)_{x}$ splits and hence $\left(i^{*} \operatorname{coker}\left(e_{1}\right)\right)_{x}$ is free. This proves (3) implies (4).

The following shows that a locally contractible matrix factorization need not be 0 in $[M F]_{\text {naive }}$.

Example 2.11. Under the assumption of Proposition [2], suppose that $\mathbb{E}=\left(\mathcal{E}_{1} \rightarrow\right.$ $\left.\mathcal{E}_{0} \rightarrow \mathcal{E}_{1}(1)\right)$ is a matrix factorization of $(X, \mathcal{L}, W)$ such that $i^{*} \mathcal{M}:=i^{*} \operatorname{coker}\left(\mathcal{E}_{1} \rightarrow\right.$ $\left.\mathcal{E}_{0}\right)$ is a locally free coherent $\mathcal{O}_{Y^{\prime}}$-sheaf, but that the surjection $i^{*} \mathcal{E}_{0} \rightarrow i^{*} \mathcal{M}$ does not split. Then by Proposition $\mathbb{Z M}, \mathbb{E}$ is locally contractible, but $\mathbb{E}$ is not isomorphic to 0 in $[M F]_{\text {naive }}$. Such examples are common. For instance, take $X=\mathbb{P}_{k}^{2}=$ Proj $k\left[T_{0}, T_{1}, T_{2}\right], \mathcal{L}=\mathcal{O}_{X}(1)$ and $W=T_{2}$, so that $Y=\mathbb{P}_{k}^{1}=\operatorname{Proj} k\left[T_{0}, T_{1}\right]$. Then let $\mathcal{M}=\mathcal{O}_{Y}, \mathcal{E}_{0}=\mathcal{O}_{X}(-1)^{2}$ and $\mathcal{E}_{0} \rightarrow i_{*} \mathcal{M}$ be the composition of

$$
\mathcal{E}_{0} \stackrel{\text { can }}{\longrightarrow} i_{*} \mathcal{O}_{Y}(-1)^{2} \stackrel{\left(T_{0}, T_{2}\right)}{\longrightarrow} i_{*} \mathcal{O}_{Y}
$$

Then the kernel $\mathcal{E}_{1}$ of $\mathcal{E}_{0} \rightarrow i_{*} \mathcal{M}$ is locally free and, using the argument found in the introduction, this leads to a matrix factorization of $(X, \mathcal{L}, W)$ with $i^{*} \operatorname{coker}\left(\mathcal{E}_{1} \rightarrow\right.$ $\left.\mathcal{E}_{0}\right)=\mathcal{M}$. But the surjection

$$
i^{*} \mathcal{E}_{0}=\mathcal{O}_{Y}(-1)^{2} \stackrel{\left(T_{0}, T_{1}\right)}{\longrightarrow} \mathcal{O}_{Y}=\mathcal{M}
$$

does not split.

The collection of locally contractible objects is the intersection of the kernels of the triangulated functors

$$
[M F(X, \mathcal{L}, W)]_{\text {naive }} \rightarrow\left[M F\left(\mathcal{O}_{X, x}, \mathcal{L}_{x}, W_{x}\right)\right],
$$

as $x$ ranges over all points of $X$. Recall that a triangulated subcategory of a triangulated category is thick if it is closed under direct summands. The kernel of any triangulated functor is thick and an arbitrary intersection of thick subcategories is thick. Thus the collection of locally contractible objects forms a thick subcategory of $[M F(X, \mathcal{L}, W)]_{\text {naive }}$.

The following category, whose definition is originally due to Orlov and appeared, for example, in [ []], is the central object of study in this paper:

Definition 2.12. The homotopy category of matrix factorizations, written $[M F(X, \mathcal{L}, W)]$, is the Verdier quotient of $[M F(X, \mathcal{L}, W)]_{\text {naive }}$ by the thick subcategory of locally contractible objects:

$$
[M F(X, \mathcal{L}, W)]=\frac{[M F(X, \mathcal{L}, W)]_{\text {naive }}}{\text { locally contractible objects }} .
$$

Remark 2.13. A strict map $\mathbb{E}^{\prime} \rightarrow \mathbb{E}$ is a weak equivalence if and only if it fits into a distinguished triangle

$$
\mathbb{E}^{\prime} \rightarrow \mathbb{E} \rightarrow \mathbb{F} \rightarrow \mathbb{E}^{\prime}[1]
$$

in $[M F(X, \mathcal{L}, W)]_{\text {naive }}$ such that $\mathbb{F}$ is locally contractible. Thus weak equivalences are invertible in $[M F(X, \mathcal{L}, W)]$. 
Example 2.14. If $X$ is affine, $\mathcal{L}=\mathcal{O}_{X}$ and $W$ is a non-zero-divisor, then

$$
[M F(X, \mathcal{L}, W)]=[M F(X, \mathcal{L}, W)]_{\text {naive }}
$$

by Proposition [प].

\section{Another version of the homotopy category}

The aim of this section is to describe another category associated to $M F(X, \mathcal{L}, W)$, which we write as $[M F(X, \mathcal{L}, W)]_{\mathbb{H}}$. In the next section, we prove that when $X$ is projective over a Noetherian ring and $\mathcal{L}$ is the corresponding very ample line bundle, $[M F]_{\mathbb{H}}$ and $[M F]$ are equivalent. The advantage $[M F]_{\mathbb{H}}$ enjoys over $[M F]$ is that its Hom sets are more explicit.

We make a fixed choice of a finite affine open cover $\mathcal{U}=\left\{U_{1}, \ldots, U_{m}\right\}$ of $X$, and for any quasi-coherent sheaf $\mathcal{F}$ on $X$, let $\Gamma(\mathcal{U}, \mathcal{F})$ denote the cochain complex given by the usual Cech construction. Since $X$ is separated, the cohomology of the complex $\Gamma(\mathcal{U}, \mathcal{F})$ gives the sheaf cohomology of $\mathcal{F}$. We define $\Gamma\left(\mathcal{U}, \underline{\operatorname{Hom}}_{\mathrm{MF}}(\mathbb{E}, \mathbb{F})\right)$ to be the total complex associated to the bicomplex

$$
0 \rightarrow \bigoplus_{i} \Gamma\left(U_{i}, \underline{\operatorname{Hom}}_{\mathrm{MF}}(\mathbb{E}, \mathbb{F})\right) \rightarrow \bigoplus_{i<j} \Gamma\left(U_{i} \cap U_{i}, \underline{\operatorname{Hom}}_{\mathrm{MF}}(\mathbb{E}, \mathbb{F})\right) \rightarrow \cdots
$$

given by applying the Cech construction degree-wise. If $\mathbb{G}$ is another matrix factorization, there is an evident morphism of chain complexes

$$
\Gamma\left(\mathcal{U}, \underline{\operatorname{Hom}}_{\mathrm{MF}}(\mathbb{E}, \mathbb{F})\right) \otimes \Gamma\left(\mathcal{U}, \underline{\operatorname{Hom}}_{\mathrm{MF}}(\mathbb{F}, \mathbb{G})\right) \rightarrow \Gamma\left(\mathcal{U}, \underline{\operatorname{Hom}}_{\mathrm{MF}}(\mathbb{E}, \mathbb{G})\right)
$$

which one can check is associative and unital. Thus $M F(X, \mathcal{L}, W)$, with function spaces $\Gamma\left(\mathcal{U}, \underline{\operatorname{Hom}}_{\mathrm{MF}}(\mathbb{E}, \mathbb{F})\right)$, is a DG category. We set

$$
\mathbb{H}^{q}\left(X, \underline{\operatorname{Hom}}_{\mathrm{MF}}(\mathbb{E}, \mathbb{F})\right)=H^{q}\left(\Gamma\left(\mathcal{U}, \underline{\operatorname{Hom}}_{\mathrm{MF}}(\mathbb{E}, \mathbb{F})\right)\right) .
$$

There is a convergent spectral sequence

$$
H^{p}\left(X, \mathcal{H}^{q}\left(\underline{\operatorname{Hom}}_{\mathrm{MF}}(\mathbb{E}, \mathbb{F})\right)\right) \Longrightarrow \mathbb{H}^{p+q}\left(X, \underline{\operatorname{Hom}}_{\mathrm{MF}}(\mathbb{E}, \mathbb{F})\right),
$$

where $\mathcal{H}^{q}$ is the $q$-th cohomology sheaf of a complex. In particular, if $\underline{\operatorname{Hom}}_{\mathrm{MF}}(\mathbb{E}, \mathbb{F}) \rightarrow$ $\underline{\operatorname{Hom}}_{\mathrm{MF}}\left(\mathbb{E}^{\prime}, \mathbb{F}^{\prime}\right)$ is a quasi-isomorphism, then the map

$$
\mathbb{H}^{n}\left(X, \underline{\operatorname{Hom}}_{\mathrm{MF}}(\mathbb{E}, \mathbb{F})\right) \rightarrow \mathbb{H}^{n}\left(X, \underline{\operatorname{Hom}}_{\mathrm{MF}}\left(\mathbb{E}^{\prime}, \mathbb{F}^{\prime}\right)\right)
$$

is an isomorphism for all $n$.

Definition 3.1. Define the category

$$
[M F(X, \mathcal{L}, W)]_{\mathbb{H}}
$$

whose objects are matrix factorizations and whose morphisms are

$$
\operatorname{Hom}_{[M F]_{H}}(\mathbb{E}, \mathbb{F})=\mathbb{H}^{0}\left(X, \underline{\operatorname{Hom}}_{\mathrm{MF}}(\mathbb{E}, \mathbb{F})\right) .
$$

Thus $[M F]_{\mathbb{H}}$ is the homotopy category associated to the DG category above.

Remark 3.2. This definition was inspired by Shipman's category of graded $D$ Branes [प]], who was in turn inspired by Segal [प]]. 
There is a canonical functor

$$
[M F(X, \mathcal{L}, W)]_{\text {naive }} \rightarrow[M F(X, \mathcal{L}, W)]_{\mathbb{H}}
$$

that is the identity on objects and is given on morphisms by the canonical map

$$
H^{0} \Gamma\left(X, \underline{\operatorname{Hom}}_{\mathrm{MF}}(\mathbb{E}, \mathbb{F})\right) \rightarrow \mathbb{H}^{0}\left(X, \underline{\operatorname{Hom}}_{\mathrm{MF}}(\mathbb{E}, \mathbb{F})\right) .
$$

Example 3.3. If $X$ is affine this functor is an equivalence since each map

$$
H^{0} \Gamma\left(X, \underline{\operatorname{Hom}}_{\mathrm{MF}}(\mathbb{E}, \mathbb{F})\right) \rightarrow \mathbb{H}^{0}\left(X, \underline{\operatorname{Hom}}_{\mathrm{MF}}(\mathbb{E}, \mathbb{F})\right)
$$

is an isomorphism. If we further assume $\mathcal{L}=\mathcal{O}_{X}$ and $W$ is a non-zero-divisor of $Q$, then both $[M F(X, \mathcal{L}, W)]_{\text {naive }}$ and $[M F(X, \mathcal{L}, W)]_{\mathbb{H}}$ are equivalent to $[M F(X, \mathcal{L}, W)]$ by Example [.].

Lemma 3.4. If a strict morphism $f: \mathbb{E}^{\prime} \rightarrow \mathbb{E}$ of matrix factorizations is a weak equivalence, then for all matrix factorizations $\mathbb{F}$, the induced map on mapping complexes

$$
\underline{\operatorname{Hom}}_{\mathrm{MF}}(\mathbb{E}, \mathbb{F}) \rightarrow \underline{\operatorname{Hom}}_{\mathrm{MF}}\left(\mathbb{E}^{\prime}, \mathbb{F}\right)
$$

is a quasi-isomorphism in $\operatorname{TPC}(X, \mathcal{L})$. In particular, the map in $[M F(X, \mathcal{L}, W)]_{\mathbb{H}}$ induced by $f: \mathbb{E}^{\prime} \rightarrow \mathbb{E}$ is an isomorphism.

Proof. For matrix factorizations $\mathbb{E}, \mathbb{F}$ and for all $x \in X$ there is an isomorphism

$$
\underline{\operatorname{Hom}}_{M F(X, \mathcal{L}, W)}(\mathbb{E}, \mathbb{F})_{x} \cong \underline{\operatorname{Hom}}_{M F\left(\mathcal{O}_{X, x}, \mathcal{L}_{x}, W_{x}\right)}\left(\mathbb{E}_{x}, \mathbb{F}_{x}\right) \text {. }
$$

Since we are assuming $\mathbb{E}_{x}^{\prime} \rightarrow \mathbb{E}_{x}$ is an isomorphism in $\left[M F\left(\mathcal{O}_{X, x}, \mathcal{L}_{x}, W_{x}\right)\right]$, it follows that

$$
\underline{\operatorname{Hom}}_{M F(X, \mathcal{L}, W)}(\mathbb{E}, \mathbb{F})_{x} \rightarrow \underline{\operatorname{Hom}}_{M F(X, \mathcal{L}, W)}\left(\mathbb{E}^{\prime}, \mathbb{F}\right)_{x}
$$

is a quasi-isomorphism for all $\mathbb{F}$ and $x$. This proves

$$
\underline{\operatorname{Hom}}_{\mathrm{MF}}(\mathbb{E}, \mathbb{F}) \rightarrow \underline{\operatorname{Hom}}_{\mathrm{MF}}\left(\mathbb{E}^{\prime}, \mathbb{F}\right)
$$

is a quasi-isomorphism in $\operatorname{TPC}(X, \mathcal{L})$ and hence that

$$
\mathbb{H}^{n}\left(X, \underline{\operatorname{Hom}}_{\mathrm{MF}}(\mathbb{E}, \mathbb{F})\right) \stackrel{\cong}{\longrightarrow} \mathbb{H}^{n}\left(X, \underline{\operatorname{Hom}}_{\mathrm{MF}}\left(\mathbb{E}^{\prime}, \mathbb{F}\right)\right)
$$

is an isomorphism for all $n$. The case $n=0$ shows $\mathbb{E}$ and $\mathbb{E}^{\prime}$ co-represent the same functor on $[M F(X, \mathcal{L}, W)]_{\mathbb{H}}$ and hence are isomorphic.

The following is a formal consequence of the lemma:

Proposition 3.5. The functor $[M F(X, \mathcal{L}, W)]_{\text {naive }} \rightarrow[M F(X, \mathcal{L}, W)]_{\mathbb{H}}$ factors canonically as

$$
[M F(X, \mathcal{L}, W)]_{\text {naive }} \rightarrow[M F(X, \mathcal{L}, W)] \rightarrow[M F(X, \mathcal{L}, W)]_{\mathbb{H}}
$$

\section{Equivalence of homotopy categories}

The goal of this section is to prove Theorem $\mathbb{\square}$ of the introduction: the functor

$$
[M F(X, \mathcal{L}, W)] \rightarrow[M F(X, \mathcal{L}, W)]_{\mathbb{H}}
$$

is an equivalence of categories when $X$ is projective over a Noetherian ring and $\mathcal{L}$ is the corresponding very ample line bundle. For such an $X$ and $\mathcal{L}$, we will often use Serre's 
Vanishing Theorem: for any coherent sheaf $\mathcal{F}$ on $X$ and all $n \gg 0, H^{i}(X, \mathcal{F}(n))=0$ for $i>0$.

Given a bounded complex of strict morphisms of matrix factorizations

$$
\mathbb{E}^{\bullet}:=\left(\mathbb{E}^{p} \rightarrow \mathbb{E}^{p+1} \rightarrow \cdots \rightarrow \mathbb{E}^{q}\right),
$$

we define its total object, written $\operatorname{Tot}\left(\mathbb{E}^{\bullet}\right)$, as follows. We may visualize $\mathbb{E}^{\bullet}$ as a commutative diagram

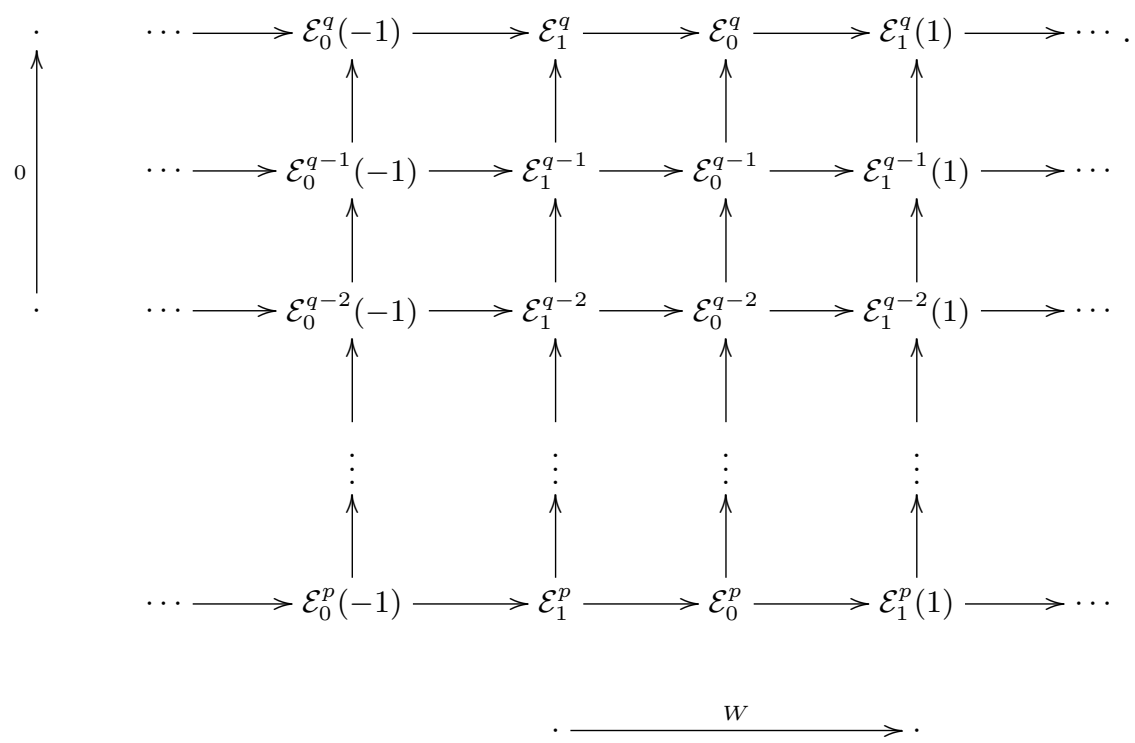

We form $\operatorname{Tot}\left(\mathbb{E}^{\bullet}\right)$ by taking direct sums along lines of slope -1 in this diagram, and maps are defined just as for the usual total complex associated to a bicomplex. The resulting chain of maps clearly satisfies the required twisted periodicity, making it an object of $M F(X, \mathcal{L}, W)$.

A special case of the Tot construction will be especially useful. First, for a locally free coherent sheaf $\mathcal{P}$ on $X$ and a matrix factorization $\mathbb{E}$, let $\mathcal{P} \otimes \mathbb{E}$ denote the matrix factorization obtained by applying the functor $\mathcal{P} \otimes_{\mathcal{O}_{X}}-$ to the data defining $\mathbb{E}$. If $\mathcal{P}^{\bullet}$ is a bounded complex of locally free coherent sheaves on $X$, then

$$
\mathcal{P}^{\bullet} \otimes \mathbb{E}=\left(\mathcal{P}^{p} \otimes \mathbb{E} \rightarrow \mathcal{P}^{p+1} \otimes \mathbb{E} \rightarrow \cdots \rightarrow \mathcal{P}^{q} \otimes \mathbb{E}\right)
$$

is a bounded complex of strict morphisms of matrix factorizations, and we may form its associated total matrix factorization $\operatorname{Tot}\left(\mathcal{P}^{\bullet} \otimes \mathbb{E}\right)$.

We will need the following lemma.

Lemma 4.1. Let $X$ be a Noetherian separated scheme, $\mathcal{L}$ any line bundle on $X$ and $W$ a global section of $\mathcal{L}$. If $\mathcal{P}_{1}^{\bullet} \rightarrow \mathcal{P}_{2}^{\bullet}$ is a quasi-isomorphism between bounded complexes of locally free coherent sheaves on $X$, then

$$
\operatorname{Tot}\left(\mathcal{P}_{1}^{\bullet} \otimes \mathbb{E}\right) \rightarrow \operatorname{Tot}\left(\mathcal{P}_{2}^{\bullet} \otimes \mathbb{E}\right)
$$

is a weak equivalence of matrix factorizations. 
Proof. We may localize at a point $x$, in which case the assertion becomes: If $W$ is an element in a local ring $Q, P_{1}^{\bullet} \rightarrow P_{2}^{\bullet}$ is a quasi-isomorphism of bounded complexes of free $Q$-modules of finite rank, and $\mathbb{E}$ is a matrix factorization of $W$ over $Q$, then

$$
\operatorname{Tot}\left(P_{1}^{\bullet} \otimes \mathbb{E}\right) \rightarrow \operatorname{Tot}\left(P_{2}^{\bullet} \otimes \mathbb{E}\right)
$$

is an isomorphism in the category $[M F(\operatorname{Spec} Q, \mathcal{O}, W)]$. In this setting, $P_{1}^{\bullet} \rightarrow P_{2}^{\bullet}$ is a chain homotopy equivalence (i.e., there is an inverse up to chain homotopy). It therefore suffices to note that the functor $\operatorname{Tot}(-\otimes \mathbb{E})$ from bounded complexes of free modules to matrix factorizations sends chain homotopies of complexes to homotopies of matrix factorizations.

Theorem 4.2. Let $X$ be a scheme that is projective over a Noetherian ring $Q$ and $\mathcal{L}=\mathcal{O}_{X}(1)$ the corresponding very ample line bundle on $X$. For any global section $W$ of $\mathcal{L}$, the canonical functor

$$
[M F(X, \mathcal{L}, W)] \rightarrow[M F(X, \mathcal{L}, W)]_{\mathbb{H}}
$$

is an equivalence.

The proof of the theorem uses the following lemma.

Lemma 4.3. Under the assumptions of Theorem 4.9 , for any pair of matrix factorizations $\mathbb{E}, \mathbb{F}$, there is a weak equivalence $\mathbb{E}^{\prime} \rightarrow \mathbb{E}$ such that the canonical map

$$
\operatorname{Hom}_{[M F]_{\text {naive }}}\left(\mathbb{E}^{\prime}, \mathbb{F}\right) \stackrel{\cong}{\longrightarrow} \operatorname{Hom}_{[M F]_{\mathbb{H}}}\left(\mathbb{E}^{\prime}, \mathbb{F}\right)
$$

is an isomorphism.

Proof. Say $X$ is a closed subscheme of $\mathbb{P}_{Q}^{m}=\operatorname{Proj} Q\left[x_{0}, \ldots, x_{m}\right]$ and $\mathcal{L}=\mathcal{O}_{X}(1)$ is the restriction of $\mathcal{O}_{\mathbb{P} m}(1)$ to $X$.

For each positive integer $j$ we have a surjection

$$
\mathcal{O}_{X}(-j)^{k} \rightarrow \mathcal{O}_{X}
$$

given by the the set of monomials of degree $j$ in $m+1$ variables (so that $k=k(j)$ is the number of such monomials). Let $\mathcal{P}(j)^{\bullet}$ be the associated "truncated" Koszul complex

$$
0 \rightarrow \mathcal{O}_{X}(-k j)^{\left(\begin{array}{l}
k \\
k
\end{array}\right)} \rightarrow \mathcal{O}_{X}(-(k-1) j)^{\left(\begin{array}{c}
k \\
k-1
\end{array}\right)} \rightarrow \cdots \rightarrow \mathcal{O}_{X}(-2 j)^{\left(\begin{array}{c}
k \\
2
\end{array}\right)} \rightarrow \mathcal{O}_{X}(-j)^{k} \rightarrow 0,
$$

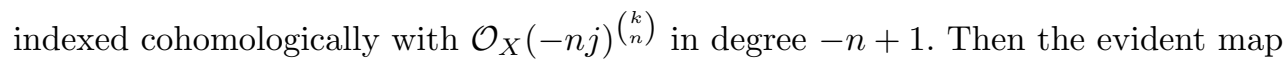

$$
\mathcal{P}(j) \stackrel{\sim}{\sim} \mathcal{O}_{X}
$$

is a quasi-isomorphism, and so by Lemma 1 , the induced map

$$
\mathbb{E}^{\prime}:=\operatorname{Tot}(\mathcal{P}(j) \bullet \mathbb{E}) \stackrel{\sim}{\longrightarrow} \operatorname{Tot}\left(\mathcal{O}_{X} \otimes \mathbb{E}\right)=\mathbb{E}
$$

is a weak equivalence. We prove that for $j \gg 0$ this weak equivalence has the desired property.

Write $\mathcal{E}_{0}, \mathcal{E}_{1}$ and $\mathcal{E}_{0}^{\prime}, \mathcal{E}_{1}^{\prime}$ for the components of $\mathbb{E}$ and $\mathbb{E}^{\prime}$. We have that

$$
\mathcal{E}_{0}^{\prime}=\mathcal{E}_{0}(-j)^{k} \oplus \mathcal{E}_{1}(1-2 j)^{\left(\begin{array}{c}
k \\
2
\end{array}\right)} \oplus \mathcal{E}_{0}(1-3 j)^{\left(\begin{array}{c}
k \\
3
\end{array}\right)} \oplus \mathcal{E}_{1}(2-4 j)^{\left(\begin{array}{c}
k \\
4
\end{array}\right)} \oplus \cdots
$$

and similarly for $\mathcal{E}_{1}^{\prime}$. In particular, for any integer $N$ we may choose $j \gg 0$ so that $\mathcal{E}_{0}^{\prime}$ and $\mathcal{E}_{1}^{\prime}$ are direct sums of locally free coherent sheaves of the form $\mathcal{E}_{0}(-a)$ and $\mathcal{E}_{1}(-b)$ with $a, b \geqslant N$. 
Let $\mathcal{C}$ denote the twisted two-periodic complex $\underline{\operatorname{Hom}}_{\mathrm{MF}}\left(\mathbb{E}^{\prime}, \mathbb{F}\right)$. By the above, for any integer $N$, we may pick $j \gg 0$ so that $\mathcal{C}^{0}$ and $\mathcal{C}^{1}$ are direct sums of locally free coherent sheaves of the form

$$
\begin{aligned}
\underline{\operatorname{Hom}}_{\mathcal{O}_{X}}\left(\mathcal{E}_{0}, \mathcal{F}_{0}\right)(a), & \underline{\operatorname{Hom}}_{\mathcal{O}_{X}}\left(\mathcal{E}_{1}, \mathcal{F}_{0}\right)(b), \underline{\operatorname{Hom}}_{\mathcal{O}_{X}}\left(\mathcal{E}_{0}, \mathcal{F}_{1}\right)(c), \\
\text { or } & \underline{\operatorname{Hom}}_{\mathcal{O}_{X}}\left(\mathcal{E}_{1}, \mathcal{F}_{1}\right)(d)
\end{aligned}
$$

with $a, b, c, d \geqslant N$. Thinking of $\mathcal{C}$ as an unbounded complex we have $\mathcal{C}^{q}=\mathcal{C}^{0}\left(\frac{q}{2}\right)$ if $q$ is even or $\mathcal{C}^{q}=\mathcal{C}^{1}\left(\frac{q-1}{2}\right)$ if $q$ is odd. Thus, for any integers $N$ and $M$, we may choose $j$ sufficiently large so that each of $\mathcal{C}^{M}, \mathcal{C}^{M+1}, \cdots$ is a direct sum of locally free coherent sheaves as in (⿴囗十) with $a, b, c, d \geqslant N$. In particular, for any $M$, we may choose $j$ sufficiently large so that $H^{p}\left(X, \mathcal{C}^{q}\right)=0$ for all $p>0$ and all $q \geqslant M$.

The result now follows from the spectral sequence

$$
E_{1}^{p, q}=H^{p}\left(X, \mathcal{C}^{q}\right) \Longrightarrow \mathbb{H}^{p+q}\left(X, \underline{\operatorname{Hom}}_{\mathrm{MF}}\left(\mathbb{E}^{\prime}, \mathbb{F}\right)\right)
$$

using that $X$ has bounded cohomological dimension for quasi-coherent sheaves. In more detail, if $X$ has cohomological dimension $n$, we may choose $j$ sufficiently large so that $H^{p}\left(X, \mathcal{C}^{q}\right)=0$ for all $p>0$ and $q \geqslant-n-1$. It follows that

$$
\mathbb{H}^{0}\left(X, \underline{\operatorname{Hom}}_{\mathrm{MF}}\left(\mathbb{E}^{\prime}, \mathbb{F}\right)\right) \cong E_{2}^{0,0}=H^{0} \Gamma\left(X, \underline{\operatorname{Hom}}_{\mathrm{MF}}\left(\mathbb{E}^{\prime}, \mathbb{F}\right)\right) .
$$

Remark 4.4. The proof of the Lemma actually shows that for any matrix factorizations $\mathbb{E}, \mathbb{F}$ and any integer $M$, there is a weak equivalence $\mathbb{E}^{\prime} \rightarrow \mathbb{E}$ so that

$$
H^{q} \Gamma\left(X, \underline{\operatorname{Hom}}_{\mathrm{MF}}\left(\mathbb{E}^{\prime}, \mathbb{F}\right)\right) \rightarrow \mathbb{H}^{q}\left(X, \underline{\operatorname{Hom}}_{\mathrm{MF}}\left(\mathbb{E}^{\prime}, \mathbb{F}\right)\right)
$$

is an isomorphism for all $q \geqslant M$.

Proof of Theorem 4.9. We need to prove

$$
\operatorname{Hom}_{[M F]}(\mathbb{E}, \mathbb{F}) \rightarrow \operatorname{Hom}_{[M F]_{\mathbb{H}}}(\mathbb{E}, \mathbb{F})
$$

is an isomorphism for every pair of matrix factorizations $\mathbb{E}, \mathbb{F}$. Given such a pair, let $\mathbb{E}^{\prime} \rightarrow \mathbb{E}$ be a weak equivalence as in Lemma 4.3 so that

$$
\operatorname{Hom}_{[M F]_{\text {naive }}}\left(\mathbb{E}^{\prime}, \mathbb{F}\right) \stackrel{\cong}{\longrightarrow} \operatorname{Hom}_{[M F]_{\mathbb{H}}}\left(\mathbb{E}^{\prime}, \mathbb{F}\right),
$$

and consider the commutative diagram

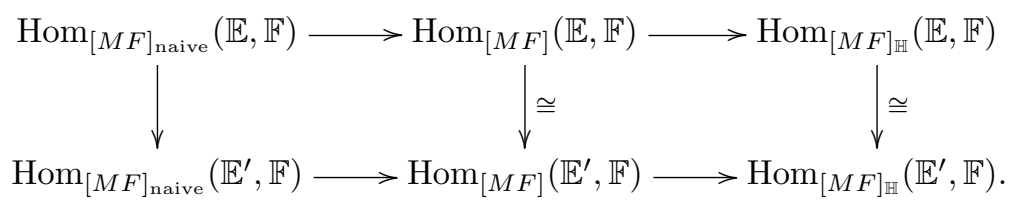

The middle and right-most vertical arrows are isomorphisms and the composition of the arrows along the bottom is an isomorphism. It follows that (回) is onto.

Suppose $\alpha \in \operatorname{Hom}_{[M F]}(\mathbb{E}, \mathbb{F})$ is in the kernel of (四). We may represent $\alpha$ by a diagram of strict morphisms

$$
\mathbb{E} \stackrel{s}{\longleftarrow} \mathbb{G} \stackrel{\beta}{\longrightarrow} \mathbb{F}
$$

with $s$ a weak equivalence. Let $\mathbb{G}^{\prime} \rightarrow \mathbb{G}$ be a weak equivalence for the pair $\mathbb{G}, \mathbb{F}$ given 
by Lemma 4.3 , so that

$$
\operatorname{Hom}_{[M F]_{\text {naive }}}\left(\mathbb{G}^{\prime}, \mathbb{F}\right) \stackrel{\cong}{\longrightarrow} \operatorname{Hom}_{[M F]_{\mathbb{H}}}\left(\mathbb{G}^{\prime}, \mathbb{F}\right)
$$

is an isomorphism. By precomposing the above diagram for $\alpha$ with the weak equivalence $\mathbb{G}^{\prime} \rightarrow \mathbb{G}$, we may represent $\alpha$ also as a diagram of strict morphisms of the form

$$
\mathbb{E} \stackrel{s^{\prime}}{\longleftarrow} \mathbb{G}^{\prime} \stackrel{\beta^{\prime}}{\longrightarrow} \mathbb{F}
$$

with $s^{\prime}$ a weak equivalence. Since $\alpha$ is mapped to zero and $s^{\prime}$ is mapped to an isomorphism, $\beta^{\prime}$ is mapped to zero in $[M F]_{\mathbb{H}}$. But then since

$$
\operatorname{Hom}_{[M F]_{\text {naive }}}\left(\mathbb{G}^{\prime}, \mathbb{F}\right) \stackrel{\cong}{\longrightarrow} \operatorname{Hom}_{[M F]_{\mathbb{H}}}\left(\mathbb{G}^{\prime}, \mathbb{F}\right)
$$

is an isomorphism, we have that $\beta^{\prime}$ is zero already in $[M F]_{\text {naive }}$ and hence also is zero in $[M F]$. It follows that $\alpha=0$ in $[M F]$.

\section{Hom-sets in the singularity category}

In this section we return to the general situation, with $X$ a Noetherian separated scheme and $\mathcal{L}$ a line bundle. But we make the further assumption that $W \in \Gamma(X, \mathcal{L})$ is a regular section, i.e., $W: \mathcal{O}_{X} \rightarrow \mathcal{L}$ is injective. Equivalently, for each $x \in X$, the element $W_{x} \in \mathcal{O}_{X, x} \cong \mathcal{L}_{x}$ is a non-zero-divisor. Define $Y$ to be the zero subscheme of $W$ (i.e., by the ideal given as the image of the injective map $W^{*}: \mathcal{L}^{*} \rightarrow \mathcal{O}_{X}$ ).

The singularity category of a scheme $Z$ is the Verdier quotient

$$
\mathrm{D}_{\mathrm{sg}}(Z):=D^{b}(Z) / \operatorname{Perf}(Z)
$$

where $D^{b}(Z)$ is the bounded derived category of coherent sheaves and $\operatorname{Perf}(Z)$ is the full subcategory consisting of perfect complexes - i.e., those complexes that are locally quasi-isomorphic to bounded complexes of free modules of finite rank. This construction was introduced by Buchweitz [ $[\mathbf{U}]$ in the case when $Z$ is affine and rediscovered by Orlov [ [5].

For a matrix factorization

$$
\mathbb{E}=\left(\mathcal{E}_{1} \stackrel{e_{1}}{\longrightarrow} \mathcal{E}_{0} \stackrel{e_{0}}{\longrightarrow} \mathcal{E}_{1}(1)\right)
$$

define coker $(\mathbb{E})$ to be coker $\left(e_{1}\right)$. Multiplication by $W$ on the coherent sheaf coker $(\mathbb{E})$ is zero and hence coker $(\mathbb{E})$ may be regarded as a coherent sheaf on $Y$ and thus as an object of $\mathrm{D}_{\mathrm{sg}}(Y)$. (More formally, the canonical map gives an isomorphism $\operatorname{coker}\left(e_{1}\right) \cong i_{*} i^{*} \operatorname{coker}\left(e_{1}\right)$ and we define $\operatorname{coker}(\mathbb{E})=i^{*} \operatorname{coker}\left(e_{1}\right)$.)

Definition 5.1. For a matrix factorization $\mathbb{E}=\left(\mathcal{E}_{1} \stackrel{e_{1}}{\longrightarrow} \mathcal{E}_{0} \stackrel{e_{0}}{\longrightarrow} \mathcal{E}_{1}(1)\right), i^{*} \mathbb{E}$ is the chain complex of locally free coherent sheaves on $Y$

$$
\cdots \rightarrow i^{*} \mathcal{E}_{0}(-1) \stackrel{e_{0}(-1)}{\longrightarrow} i^{*} \mathcal{E}_{1} \stackrel{e_{1}}{\longrightarrow} i^{*} \mathcal{E}_{0} \stackrel{e_{0}}{\longrightarrow} i^{*} \mathcal{E}_{1}(1) \stackrel{e_{1}(1)}{\longrightarrow} i^{*} \mathcal{E}_{0}(1) \rightarrow \cdots
$$

We write $\underline{\operatorname{Hom}}_{\mathcal{O}_{Y}}(\mathcal{M}, \mathcal{N})$ for the sheaf of homomorphisms between two sheaves $\mathcal{M}, \mathcal{N}$ on $Y$ (or the total product complex of the bicomplex of such if $\mathcal{M}$ or $\mathcal{N}$ is a complex) and $\underline{\operatorname{Ext}}_{\mathcal{O}_{Y}}^{*}(\mathcal{M}, \mathcal{N})$ for the right derived functors of $\underline{\operatorname{Hom}}_{\mathcal{O}_{Y}}(\mathcal{M},-)$. 
Lemma 5.2. Assume $X$ is a Noetherian separated scheme, $\mathcal{L}$ is a line bundle on $X$, and $W$ is a regular global section of $\mathcal{L}$. Let $\mathbb{E}$ be a matrix factorization of $(X, \mathcal{L}, W)$, set $\mathcal{M}=$ coker $\mathbb{E}$, and let $\mathcal{N}$ be any coherent sheaf on $Y$. The following hold:

(1) $i^{*} \mathbb{E}$ is an acyclic complex and the brutal truncation $\left(i^{*} \mathbb{E}\right)_{\geqslant 0}$ is a resolution of $\mathcal{M}$ by locally free coherent sheaves on $Y$.

(2) For all $q \geqslant 1$, there is an isomorphism

$$
\underline{\operatorname{Ext}}_{\mathcal{O}_{Y}}^{q}(\mathcal{M}, \mathcal{N})(1) \cong \underline{\operatorname{Ext}}_{\mathcal{O}_{Y}}^{q+2}(\mathcal{M}, \mathcal{N}) .
$$

(3) If $\mathcal{N}=\operatorname{coker}(\mathbb{F})$ for a matrix factorization $\mathbb{F}$, then there is a quasi-isomorphism, natural in both $\mathbb{E}$ and $\mathbb{F}$,

$$
\underline{\operatorname{Hom}}_{\mathrm{MF}}(\mathbb{E}, \mathbb{F}) \rightarrow i_{*} \underline{\operatorname{Hom}}_{\mathcal{O}_{Y}}\left(i^{*} \mathbb{E}, \mathcal{N}\right) .
$$

(4) If $X$ is projective over a ring and $\mathcal{L}$ is the corresponding very ample line bundle, then the edge map of the local-to-global spectral sequence

$$
\operatorname{Ext}_{\mathcal{O}_{Y}}^{q}(\mathcal{M}, \mathcal{N}) \rightarrow \Gamma\left(Y, \underline{\operatorname{Ext}}_{\mathcal{O}_{Y}}^{q}(\mathcal{M}, \mathcal{N})\right)
$$

is an isomorphism for $q \gg 0$. (Here $\mathcal{N}$ can be an arbitrary coherent sheaf.)

Proof. It is clear that $i^{*} \mathbb{E}$ is a complex, since the composition of two adjacent maps is multiplication by the image of $W$ in $\mathcal{O}_{Y}(1)$, which is zero. Acyclicity may be checked locally, and for any point $x \in X$, the complex $\left(i^{*} \mathbb{E}\right)_{x}$ is a two-periodic complex induced by a matrix factorization over $\mathcal{O}_{X, x}$ of a non-zero divisor, and hence is acyclic by [ [2], 5.1]. The rest of part (1) now follows directly since $\mathcal{M}$ was defined to be $i^{*} \operatorname{coker}\left(e_{1}\right)$.

For part (2), there is an isomorphism $i^{*} \mathbb{E}(-1) \cong i^{*} \mathbb{E}[-2]$, and hence, using part (1), we have

$$
\begin{aligned}
\underline{\operatorname{Ext}}_{\mathcal{O}_{Y}}^{q}(\mathcal{M}, \mathcal{N})(1) \cong \mathcal{H}^{q}\left(\underline{\operatorname{Hom}}_{\mathcal{O}_{Y}}\left(i^{*} \mathbb{E}, \mathcal{N}\right)\right)(1) \cong \mathcal{H}^{q}\left(\underline{\operatorname{Hom}}_{\mathcal{O}_{Y}}\left(i^{*} \mathbb{E}(-1), \mathcal{N}\right)\right) \\
\cong \mathcal{H}^{q}\left(\underline{\operatorname{Hom}}_{\mathcal{O}_{Y}}\left(i^{*} \mathbb{E}[-2], \mathcal{N}\right)\right) \cong \mathcal{H}^{q+2}\left(\underline{\operatorname{Hom}}_{\mathcal{O}_{Y}}\left(i^{*} \mathbb{E}, \mathcal{N}\right)\right) \cong \underline{\operatorname{Ext}}_{\mathcal{O}_{Y}}^{q+2}(\mathcal{M}, \mathcal{N}),
\end{aligned}
$$

for $q \geqslant 1$.

For part (3), there is a surjective map of chain complexes,

$$
\underline{\operatorname{Hom}}_{\mathrm{MF}}(\mathbb{E}, \mathbb{F}) \rightarrow \underline{\operatorname{Hom}}_{\mathcal{O}_{X}}\left(\mathbb{E}, i_{*} \mathcal{N}\right),
$$

which is natural in both variables, given by the diagram

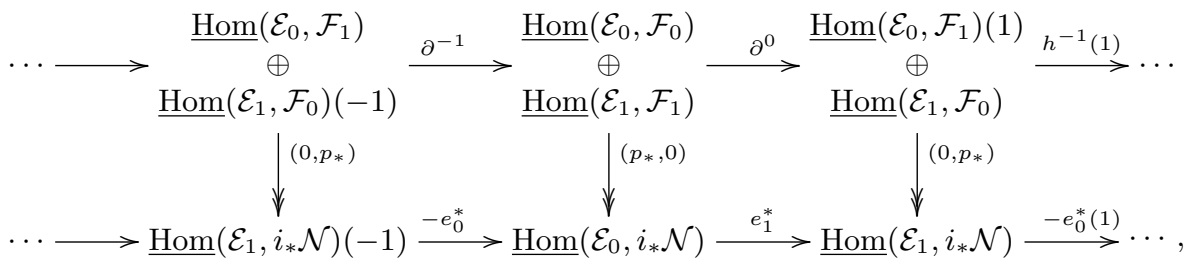

where $p: \mathcal{F}_{0} \rightarrow i_{*} \mathcal{N}$ is the canonical map. (Recall that

$$
\partial^{-1}=\left[\begin{array}{cc}
\left(f_{1}\right)_{*} & -e_{0}^{*} \\
-e_{1}^{*} & \left(f_{0}\right)_{*}
\end{array}\right] \quad \text { and } \quad \partial^{0}=\left[\begin{array}{cc}
\left(f_{0}\right)_{*} & e_{0}^{*} \\
e_{1}^{*} & \left(f_{1}\right)_{*}
\end{array}\right] \text {.) }
$$

Since $\underline{\operatorname{Hom}}_{\mathcal{O}_{X}}\left(\mathbb{E}, i_{*} \mathcal{N}\right)$ is canonically isomorphic to $i_{*} \underline{\operatorname{Hom}}_{\mathcal{O}_{Y}}\left(i^{*} \mathbb{E}, \mathcal{N}\right)$, it suffices to

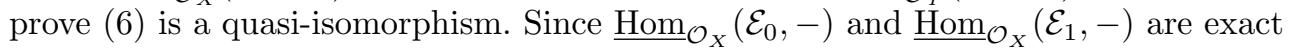


functors and $0 \rightarrow \mathcal{F}_{1} \rightarrow \mathcal{F}_{0} \rightarrow i_{*} \mathcal{N} \rightarrow 0$ is an exact sequence, the kernel of (四) is

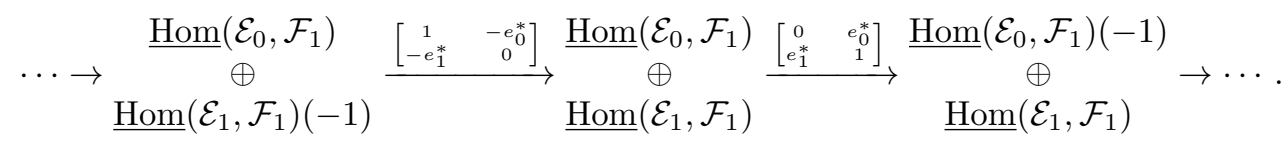

The maps

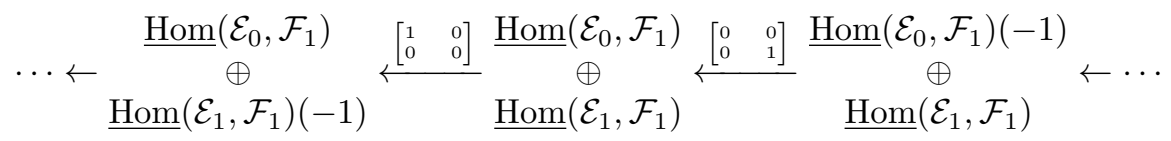

determine a contracting homotopy for this kernel, proving that (四) is a quasi-isomorphism.

For part (4), consider the local-to-global spectral sequence

$$
E_{2}^{p, q}=H^{p}\left(Y, \underline{\operatorname{Ext}}_{\mathcal{O}_{Y}}^{q}(\mathcal{M}, \mathcal{N})\right) \Longrightarrow \operatorname{Ext}_{\mathcal{O}_{Y}}^{p+q}(\mathcal{M}, \mathcal{N}) .
$$

Since $\operatorname{Ext}_{\mathcal{O}_{Y}}^{2 q+2}(\mathcal{M}, \mathcal{N}) \cong \underline{\operatorname{Ext}}_{\mathcal{O}_{Y}}^{2}(\mathcal{M}, \mathcal{N})(q)$ for all $q \geqslant 0$ and similarly for odd indices, the spectral sequence degenerates for $q \gg 0$ by Serre's Vanishing Theorem.

Recall from that a twisted periodic complex of sheaves is a complex of locally free coherent sheaves $\mathcal{C}$ together with a specified isomorphism $\alpha: \mathcal{C}[2] \cong \mathcal{C}(1)$.

Lemma 5.3. Let $Y$ be any scheme that is projective over a Noetherian ring and $\mathcal{L}=\mathcal{O}_{Y}(1)$ the corresponding very ample line bundle. (We have in mind the case when $Y \hookrightarrow X$ is the zero subscheme of $W$.) Let $\mathcal{C}$ be a twisted periodic complex on $Y$. For $i \gg 0$ there are isomorphisms

$$
H^{i} \Gamma(Y, \mathcal{C}) \cong \Gamma\left(Y, \mathcal{H}^{i}(\mathcal{C})\right)
$$

where $\Gamma(Y, \mathcal{C})$ is $\Gamma(Y,-)$ applied degree-wise to $\mathcal{C}$, and $\mathcal{H}^{i}$ is the ith cohomology sheaf of $\mathcal{C}$.

Proof. Let $\mathcal{B}^{i}$ and $\mathcal{Z}^{i}$ be the image and kernel sheaves of $\partial_{\mathcal{C}}^{i}$, respectively.

Since $\mathcal{Z}^{i} \cong \mathcal{Z}^{i-2}(1), \mathcal{B}^{i} \cong \mathcal{B}^{i-2}(1)$ and $\mathcal{L}$ is very ample, the higher sheaf cohomology groups of $\mathcal{Z}^{i}, \mathcal{B}^{i}$ vanish for $i \gg 0$ by Serre Vanishing. It follows that, for $i \gg 0$, the exact sequences

$$
0 \rightarrow \mathcal{Z}^{i} \rightarrow \mathcal{C}^{i} \stackrel{\partial_{\mathcal{C}}^{i}}{\longrightarrow} \mathcal{B}^{i} \rightarrow 0 \text { and } 0 \rightarrow \mathcal{B}^{i} \rightarrow \mathcal{Z}^{i+1} \rightarrow \mathcal{H}^{i+1} \rightarrow 0
$$

remain exact upon applying $\Gamma(-, Y)$ and thus

$$
\Gamma\left(Y, \mathcal{H}^{i+1}\right) \cong \frac{\Gamma\left(Y, \mathcal{Z}^{i+1}\right)}{\Gamma\left(Y, \mathcal{B}^{i}\right)} \cong \frac{\operatorname{ker} \Gamma\left(Y, \partial_{\mathcal{C}}^{i+1}\right)}{\operatorname{Im} \Gamma\left(Y, \partial_{\mathcal{C}}^{i}\right)}=H^{i+1} \Gamma(Y, \mathcal{C})
$$

Lemma 5.4. Assume $X$ is a Noetherian separated scheme with enough locally frees (i.e., every coherent sheaf on $X$ is the quotient of a locally free coherent sheaf), $\mathcal{L}$ is a line bundle, and $W$ is a regular global section of $\mathcal{L}$. Let $\mathbb{E}$ be a matrix factorization, let $Y \hookrightarrow X$ be the zero subscheme of $W$, and let $\mathcal{N}$ be any coherent sheaf on $Y$. The map induced by the canonical functor $D^{b}(Y) \rightarrow \mathrm{D}_{\mathrm{sg}}(Y)$

$$
\operatorname{Hom}_{D^{b}(Y)}(\operatorname{coker} \mathbb{E}, \mathcal{N}[m]) \rightarrow \operatorname{Hom}_{D_{\mathrm{sg}}(Y)}(\operatorname{coker} \mathbb{E}, \mathcal{N}[m])
$$

is an isomorphism for $m \gg 0$. 
Proof. We adapt the proof of [ [1, 1.21]. (We cannot apply this result directly since $Y$ need not be Gorenstein.)

We first show $(\mathbb{G})$ is onto for $m \gg 0$. An element of $\operatorname{Hom}_{\mathrm{D}_{\mathrm{sg}}(Y)}(\operatorname{coker} \mathbb{E}, \mathcal{N}[m])$ is represented by a diagram in $D^{b}(Y)$

$$
\operatorname{coker}(\mathbb{E}) \stackrel{s}{\leftarrow} A \stackrel{f}{\rightarrow} \mathcal{N}[m]
$$

such that cone $(s)$ is perfect. The first step is to show that we can replace $A$ by a coherent sheaf that is the cokernel of a matrix factorization.

By Lemma (1) the sheaf coker $\mathbb{E}$ admits a right resolution by locally free coherent sheaves on $Y$ :

$$
0 \rightarrow \operatorname{coker} \mathbb{E} \rightarrow Q^{1} \rightarrow Q^{2} \rightarrow \ldots
$$

where $Q^{1}=i^{*} \mathcal{E}_{1}(1), Q^{2}=i^{*} \mathcal{E}_{0}(1), \ldots$. For any $k \geqslant 1$ we thus have a distinguished triangle in $D^{b}(Y)$

$$
\mathcal{F}_{k}[-k-1] \rightarrow \operatorname{coker}(\mathbb{E}) \rightarrow Q^{\leqslant k} \rightarrow \mathcal{F}_{k}[-k]
$$

where $\mathcal{F}_{k}=\operatorname{coker}\left(Q^{k} \rightarrow Q^{k+1}\right)$. We claim there is an integer $k_{0}$ such that for $k \geqslant k_{0}$, the composition $\mathcal{F}_{k}[-k-1] \rightarrow \operatorname{coker}(\mathbb{E}) \rightarrow \operatorname{cone}(s)$ is zero. Since $\mathcal{F}_{k}$ is the cokernel of a matrix factorization, namely $\mathcal{F}_{k}=\operatorname{coker}(\mathbb{E}[k])$, this claim follows from the more general claim: given a perfect complex $\mathcal{P}^{\bullet}=\ldots \rightarrow \mathcal{P}^{n} \rightarrow \mathcal{P}^{n+1} \rightarrow \ldots$ there is an integer $k_{0}$ such that $\operatorname{Hom}_{D^{b}}\left(\operatorname{coker}(\mathbb{F})[-k-1], \mathcal{P}^{\bullet}\right)=0$ for all matrix factorizations $\mathbb{F}$ and all integers $k \geqslant k_{0}$. To prove this, observe first that, since $X$ has enough locally frees, we may assume $\mathcal{P}^{\bullet}$ is a bounded complex of locally free coherent sheaves. Now observe that $\operatorname{Ext}^{j}\left(\operatorname{coker}(\mathbb{F}), \mathcal{O}_{Y}\right)=0$ for all $j>0$; this is a local assertion and locally it is the statement that the transpose of a matrix factorization is also a matrix factorization. It follows that

$$
\underline{\operatorname{Ext}}_{Y}^{j}\left(\operatorname{coker} \mathbb{F}, \mathcal{P}^{n}\right) \cong \underline{\operatorname{Ext}}_{Y}^{j}\left(\operatorname{coker} \mathbb{F}, \mathcal{O}_{Y}\right) \otimes_{\mathcal{O}_{Y}} \mathcal{P}^{n}=0
$$

for all $j>0$ and all $n$, and then a standard argument gives the claim. (See the proof of [ [5, 1.18].)

Consider the triangle $A \stackrel{s}{\rightarrow} \operatorname{coker}(\mathbb{E}) \rightarrow$ cone $s \rightarrow A[1]$. Since for $k \geqslant k_{0}$ the composition $\mathcal{F}_{k}[-k-1] \rightarrow \operatorname{coker}(\mathbb{E}) \rightarrow \operatorname{cone}(s)$ is zero, there exists a map $g: \mathcal{F}_{k}[-k-1] \rightarrow$ $A$ that makes the left triangle commute:

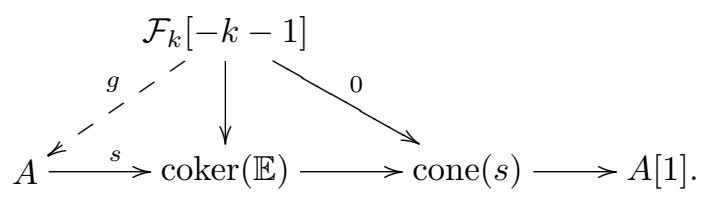

Since $\operatorname{cone}\left(\mathcal{F}_{k}[-k-1] \rightarrow \operatorname{coker}(\mathbb{E})\right)=Q^{\leqslant k}$ is perfect, composing with $g$ shows that the element of $\operatorname{Hom}_{\mathrm{D}_{\mathrm{sg}}(Y)}(\operatorname{coker} \mathbb{E}, \mathcal{N}[m])$ represented by $(\mathbb{\mathbb { Z }})$ is also represented by a diagram of the form

$$
\operatorname{coker}(\mathbb{E}) \leftarrow \mathcal{F}_{k}[-k-1] \stackrel{f \circ g}{\longrightarrow} \mathcal{N}[m]
$$

for $k \geqslant k_{0}$. 
Now consider the triangle $Q^{\leqslant k}[-1] \rightarrow \mathcal{F}_{k}[-k-1] \rightarrow \operatorname{coker}(\mathbb{E}) \rightarrow Q^{\leqslant k}$. For any $k$, there is an integer $m_{0}=m_{0}(k)$ such that

$$
\operatorname{Hom}_{D^{b}(Y)}\left(Q^{\leqslant k}[-1], \mathcal{N}[m]\right)=0
$$

for all $m \geqslant m_{0}$ - this holds because $\operatorname{Ext}^{i}\left(Q^{t}, \mathcal{N}\right) \cong H^{i}\left(Y,\left(Q^{t}\right)^{*} \otimes \mathcal{N}\right)=0$ for all $t$ and all $i \gg 0$. It follows that the composition of $Q^{\leqslant k}[-1] \rightarrow \mathcal{F}_{k}[-k-1] \stackrel{f \circ g}{\longrightarrow} \mathcal{N}[m]$ is zero for $m \geqslant m_{0}$ and hence the map $\mathcal{F}_{k}[-k-1] \rightarrow \mathcal{N}[m]$ factors through $\mathcal{F}_{k}[-k-1] \rightarrow$ $\operatorname{coker}(\mathbb{E})$. The element represented by $(\boldsymbol{\nabla})$ is thus actually represented by a map in $D^{b}(Y)$. We have proven that (घ) is onto for $k \geqslant k_{0}$ and $m \geqslant m_{0}(k)$.

Suppose now $f: \operatorname{coker}(\mathbb{E}) \rightarrow \mathcal{N}[m]$ is a morphism in $D^{b}(Y)$ that determines the zero map in $\mathrm{D}_{\mathrm{sg}}(Y)$. Then there is a map $s: A \rightarrow \operatorname{coker}(\mathbb{E})$ such that cone $(s)$ is perfect and such that $A \rightarrow \operatorname{coker}(\mathbb{E}) \stackrel{f}{\rightarrow} \mathcal{N}[m]$ is the zero map - i.e., the image of $f$ in $\mathrm{D}_{\mathrm{sg}}(Y)$ is represented by a diagram of the form $\operatorname{coker}(\mathbb{E}) \stackrel{s}{\leftarrow} A \stackrel{0}{\rightarrow} \mathcal{N}[m]$. The argument above shows that there is an integer $k_{0}$ such that we may take $A=\mathcal{F}_{k}[-k-1]$ and cone $(s)=Q^{\leqslant k}$ for $k \geqslant k_{0}$. In other words, for $k \geqslant k_{0}$, the map $f$ factors as $\operatorname{coker}(\mathbb{E}) \rightarrow Q^{\leqslant k} \rightarrow \mathcal{N}[m]$ in $D^{b}(Y)$. But, as shown above, for $m \geqslant m_{0}(k)$, we have $\operatorname{Hom}_{D^{b}(Y)}\left(Q^{\leqslant k}, \mathcal{N}[m]\right)=0$. This proves that $(\boldsymbol{\square})$ is one-to-one for $m \gg 0$.

Remark 5.5. Any scheme $X$ that is projective over a $\operatorname{ring} Q$ will have enough locally free sheaves. Indeed, we may assume that $X=\mathbb{P}_{Q}^{m}=\operatorname{Proj} Q\left[T_{1}, \ldots, T_{m}\right]$ for some ring $Q$ and $m \geqslant 0$. Then any coherent sheaf $\mathcal{F}$ is isomorphic to $\widetilde{M}$ for some finitely generated graded $Q\left[T_{1}, \ldots, T_{m}\right]$-module $M$. There exists a surjection $E \rightarrow M$ with $E$ a finitely generated graded free $Q\left[T_{1}, \ldots, T_{c}\right]$-module, and the associated map $\widetilde{E} \rightarrow \widetilde{M}$ gives the required surjection from a locally free coherent sheaf on $X$ onto $\mathcal{F}$.

For the Lemma below, it may help to keep in mind the case when $\mathcal{N}$ is the cokernel of a matrix factorization $\mathbb{F}$. In this case, by Lemma $\mathbb{Z}(3)$ we have that

$$
H^{m} \Gamma\left(Y, \underline{\operatorname{Hom}}_{\mathcal{O}_{Y}}\left(i^{*} \mathbb{E}, \mathcal{N}\right)\right) \cong H^{m} \Gamma\left(X, \underline{\operatorname{Hom}}_{M F}(\mathbb{E}, \mathbb{F})\right),
$$

and the right hand side is the set of strict morphisms between $\mathbb{E}[-m]$ and $\mathbb{F}$.

Lemma 5.6. Let $X$ be a scheme that is projective over a Noetherian ring, $\mathcal{L}=\mathcal{O}_{X}(1)$ the corresponding very ample line bundle, and $W$ a regular global section of $\mathcal{L}$. Let $\mathbb{E}$ be a matrix factorization, let $Y \hookrightarrow X$ be the zero subscheme of $W$, and let $\mathcal{N}$ be any coherent sheaf on $Y$. For every $m \in \mathbb{Z}$, there is a map, natural with respect to $\mathbb{E}$ and $\mathcal{N}$

$$
H^{m} \Gamma\left(Y, \underline{\operatorname{Hom}}_{\mathcal{O}_{Y}}\left(i^{*} \mathbb{E}, \mathcal{N}\right)\right) \rightarrow \operatorname{Hom}_{\mathrm{D}_{\mathrm{sg}}(Y)}((\operatorname{coker} \mathbb{E})[-m], \mathcal{N})
$$

that is an isomorphism for $m \gg 0$.

Proof. Any element of $H^{m} \Gamma\left(Y, \underline{\operatorname{Hom}}_{\mathcal{O}_{Y}}\left(i^{*} \mathbb{E}, \mathcal{N}\right)\right)$ gives a morphism of sheaves

$$
\operatorname{coker}(\mathbb{E}[-m]) \rightarrow \mathcal{N}
$$

which we consider as a morphism in $\mathrm{D}_{\mathrm{sg}}(Y)$. By $[\mathbf{7}, 3.12]$, there is a functorial isomorphism $\operatorname{coker}(\mathbb{E}[-m]) \cong(\operatorname{coker} \mathbb{E})[-m]$ in $\mathrm{D}_{\text {sg }}(Y)$. This gives the map. 
For $m \gg 0$ we have the following chain of isomorphisms:

$$
\begin{gathered}
\operatorname{Hom}_{D^{b}(Y)}(\operatorname{coker} \mathbb{E}[-m], \mathcal{N}) \cong \operatorname{Ext}_{\mathcal{O}_{Y}}^{m}(\operatorname{coker} \mathbb{E}, \mathcal{N}) \cong \Gamma\left(Y, \underline{\operatorname{Ext}}_{\mathcal{O}_{Y}}^{m}(\operatorname{coker} \mathbb{E}, \mathcal{N})\right) \\
\cong \Gamma\left(Y, \mathcal{H}^{m}\left(\underline{\operatorname{Hom}}_{\mathcal{O}_{Y}}\left(i^{*} \mathbb{E}, \mathcal{N}\right)\right)\right) \cong H^{m} \Gamma\left(Y, \underline{\operatorname{Hom}}_{\mathcal{O}_{Y}}\left(i^{*} \mathbb{E}, \mathcal{N}\right)\right)
\end{gathered}
$$

The second is given by 2 (4), the third by $\mathbf{2}(1)$, and the fourth by check that the diagram

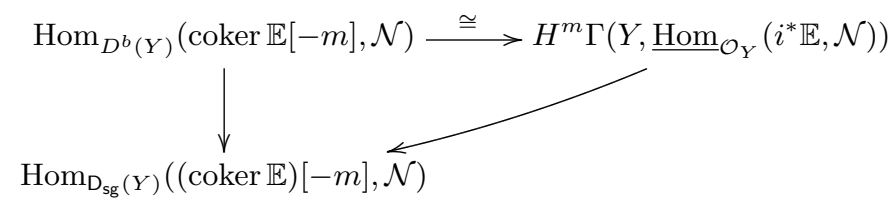

commutes, where the vertical map is induced by the canonical functor $D^{b}(Y) \rightarrow$ $\mathrm{D}_{\mathrm{sg}}(Y)$ and the diagonal map is defined above. The result now follows from Lemma [.4, which shows that the vertical arrow is an isomorphism for $m \gg 0$.

Theorem 5.7. Let $X$ be a scheme that is projective over a Noetherian ring, $\mathcal{L}=$ $\mathcal{O}_{X}(1)$ the corresponding very ample line bundle, and $W$ a regular global section of $\mathcal{L}$. Let $\mathbb{E}$ be a matrix factorization, let $Y \hookrightarrow X$ be the zero subscheme of $W$, and let $\mathcal{N}$ be any coherent sheaf on $Y$. For every $m \in \mathbb{Z}$ there is an isomorphism,

$$
\mathbb{H}^{m}\left(Y, \underline{\operatorname{Hom}}_{\mathcal{O}_{Y}}\left(i^{*} \mathbb{E}, \mathcal{N}\right)\right) \stackrel{\cong}{\rightarrow} \operatorname{Hom}_{\mathrm{D}_{\mathrm{sg}}(Y)}(\operatorname{coker} \mathbb{E}[-m], \mathcal{N})
$$

that is natural in both $\mathbb{E}$ and $\mathcal{N}$, and makes the following diagram commute:

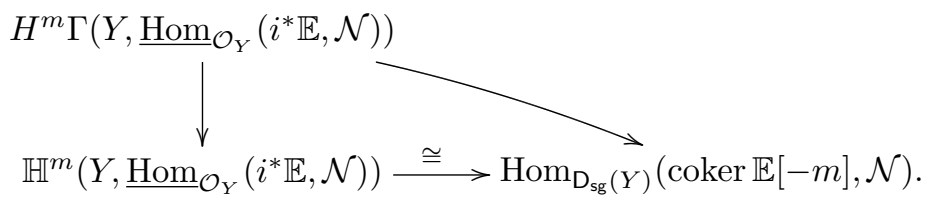

The vertical map in the diagram is the canonical one and the diagonal map is the map defined in Lemma 5.6 .

Proof. Consider the vertical and diagonal maps in $(\boldsymbol{\mathbb { U }})$, both of which are natural in both arguments. By Lemma the diagonal map is an isomorphism for $m \gg 0$. For the vertical map, we use the spectral sequence

$$
E_{2}^{p, q}=H^{p}\left(Y, \mathcal{H}^{q}\left(\underline{\operatorname{Hom}}_{\mathcal{O}_{Y}}\left(i^{*} \mathbb{E}, \mathcal{N}\right)\right)\right) \Longrightarrow \mathbb{H}^{p+q}\left(X, \underline{\operatorname{Hom}}_{\mathcal{O}_{Y}}\left(i^{*} \mathbb{E}, \mathcal{N}\right)\right)
$$

Since $\mathbb{E}$ is a matrix factorization, we have that

$$
\mathcal{H}^{2 q+2}\left(\underline{\operatorname{Hom}}_{\mathcal{O}_{Y}}\left(i^{*} \mathbb{E}, \mathcal{N}\right)\right) \cong \mathcal{H}^{2}\left(\underline{\operatorname{Hom}}_{\mathcal{O}_{Y}}\left(i^{*} \mathbb{E}, \mathcal{N}\right)\right)(q)
$$

and similarly in the odd case. Thus by Serre's Vanishing Theorem, the spectral sequence above degenerates for $q \gg 0$, giving isomorphisms

$$
\Gamma\left(Y, \mathcal{H}^{q}\left(\underline{\operatorname{Hom}}_{\mathcal{O}_{Y}}\left(i^{*} \mathbb{E}, \mathcal{N}\right)\right)\right) \cong \mathbb{H}^{q}\left(Y, \underline{\operatorname{Hom}}_{\mathcal{O}_{Y}}\left(i^{*} \mathbb{E}, \mathcal{N}\right)\right) .
$$

By Lemma 2.3, for $q \gg 0$, there is an isomorphism

$$
\Gamma\left(Y, \mathcal{H}^{q}\left(\underline{\operatorname{Hom}}_{\mathcal{O}_{Y}}\left(i^{*} \mathbb{E}, \mathcal{N}\right)\right)\right) \cong H^{q}\left(\Gamma\left(Y, \underline{\operatorname{Hom}}_{\mathcal{O}_{Y}}\left(i^{*} \mathbb{E}, \mathcal{N}\right)\right)\right)
$$

which shows that the left-hand map in (四) is an isomorphism for $q \gg 0$. 
We have proven that there is an integer $M$ such that there exists an isomorphism

$$
\mathbb{H}^{m}\left(\underline{\operatorname{Hom}}_{\mathcal{O}_{Y}}\left(i^{*} \mathbb{E}, \mathcal{N}\right)\right) \rightarrow \operatorname{Hom}_{D_{\mathrm{sg}}(Y)}(\operatorname{coker} \mathbb{E}[-m], \mathcal{N})
$$

causing (四) to commute, for all $m \geqslant M$. Since $X$ is a subscheme of Proj $Q\left[x_{1}, \ldots, x_{n}\right]$ for some Noetherian ring $Q$ and integer $n$, we have an associated Koszul exact sequence

$$
0 \rightarrow \mathcal{O}_{X} \rightarrow \mathcal{O}_{X}(1)^{n} \rightarrow \mathcal{O}_{X}(2)^{\left(\begin{array}{l}
n \\
2
\end{array}\right)} \rightarrow \cdots \rightarrow \mathcal{O}_{X}(n)^{\left(\begin{array}{l}
n \\
n
\end{array}\right)} \rightarrow 0 .
$$

Define $\mathcal{P}_{j}$ for $j=1, \ldots, n$ to be the kernel of the map $\mathcal{O}_{X}(j)^{\left(\begin{array}{c}n \\ j\end{array}\right)} \rightarrow \mathcal{O}_{X}(j+1)^{\left(\begin{array}{c}n \\ j+1\end{array}\right)}$ in this sequence. We have an exact sequence of locally free sheaves

$$
0 \rightarrow \mathcal{P}_{j} \rightarrow \mathcal{O}(j)^{\left(\begin{array}{l}
n \\
j
\end{array}\right)} \rightarrow \mathcal{P}_{j+1} \rightarrow 0
$$

for each $j=1, \ldots, n$, from which we obtain an exact sequence of matrix factorizations

$$
0 \rightarrow \mathbb{E} \otimes \mathcal{P}_{j} \rightarrow \mathbb{E} \otimes \mathcal{O}(j)^{\left(\begin{array}{c}
n \\
j
\end{array}\right)} \rightarrow \mathbb{E} \otimes \mathcal{P}_{j+1} \rightarrow 0 .
$$

We claim there exists an isomorphism

$$
\mathbb{H}^{0} \underline{\operatorname{Hom}}_{\mathcal{O}_{Y}}\left(\left(i^{*} \mathbb{E} \otimes \mathcal{P}_{j}\right)[-m], \mathcal{N}\right) \rightarrow \operatorname{Hom}_{\mathrm{D}_{\mathrm{sg}}(Y)}\left(\operatorname{coker} \mathbb{E}[-m] \otimes \mathcal{P}_{j}, \mathcal{N}\right)
$$

for all $m \geqslant M-2 j$ and for each $j=1, \ldots, n$, making the evident analogue of (四) commute. To see this, note that $\left(i^{*} \mathbb{E}\right)(j) \cong\left(i^{*} \mathbb{E}\right)[2 j]$. Thus there exists an isomorphism

$$
\mathbb{H}^{0} \underline{\operatorname{Hom}}_{\mathcal{O}_{Y}}\left(\left(\mathbb{E} \otimes \mathcal{O}(j)^{\left(\begin{array}{l}
n \\
j
\end{array}\right)}\right)[-m], \mathcal{N}\right) \rightarrow \operatorname{Hom}_{\mathrm{D}_{\mathrm{sg}}(Y)}\left(\left(\operatorname{coker} \mathbb{E} \otimes \mathcal{O}(j)^{\left(\begin{array}{c}
n \\
j
\end{array}\right)}\right)[-m], \mathcal{N}\right)
$$

for all $m \geqslant M-2 j$, making the evident analogue of (四) commute. The claim follows immediately by descending induction on $j$.

But $\mathcal{P}_{1}=\mathcal{O}_{X}$ and so $i^{*} \mathbb{E} \otimes \mathcal{P}_{1}=i^{*} \mathbb{E}$, from which we deduce that isomorphisms as in (四) exist for all $m \geqslant M-2$, having started from the assumption that there existed isomorphisms for $m \geqslant M$. Clearly such isomorphisms exist then for all $m$.

\section{Relating matrix factorizations and the singularity category}

The goal of this section is to prove Theorem $\square$ of the introduction. We continue to assume $X$ is a Noetherian separated scheme, $\mathcal{L}$ is a line bundle on $X$, and $W \in \Gamma(X, \mathcal{L})$ is a global section. Define $Y \hookrightarrow X$ to be the zero subscheme of $W$.

For a matrix factorization

$$
\mathbb{E}=\left(\mathcal{E}_{1} \stackrel{e_{1}}{\longrightarrow} \mathcal{E}_{0} \stackrel{e_{0}}{\longrightarrow} \mathcal{E}_{1}(1)\right),
$$

we view $\operatorname{coker}(\mathbb{E}):=i^{*} \operatorname{coker}\left(e_{1}\right)$ as an object of $\mathrm{D}_{\mathrm{sg}}(Y)$. This assignment is natural in $\mathbb{E}$, so gives a functor

$$
\text { coker: } \begin{aligned}
{[M F(X, \mathcal{L}, W)]_{\text {naive }} } & \rightarrow \mathrm{D}_{\mathrm{sg}}(Y) \\
\mathbb{E} & \mapsto \operatorname{coker}(\mathbb{E}) .
\end{aligned}
$$

By [0, 3.12] this is a triangulated functor.

Lemma 6.1. For a Noetherian separated scheme $X$, line bundle $\mathcal{L}$, and a regular global section $W$ of $\mathcal{L}$, if $\mathbb{E}$ is a locally contractible matrix factorization, then $\operatorname{coker}(\mathbb{E})=0$ in $\mathrm{D}_{\mathrm{sg}}(Y)$. 
Proof. It is enough to show that $\operatorname{coker}(\mathbb{E})_{x}$ is a free $\mathcal{O}_{Y, x}$ module for all $x \in Y$. By assumption $\mathbb{E}_{x}=0 \in H M F\left(\mathcal{O}_{X, x}, W_{x}\right)$ for all $x \in X$, and so the result follows from Proposition [2].

By the universal property of localization we immediately obtain:

Proposition 6.2. For a Noetherian separated scheme $X$, a line bundle $\mathcal{L}$ and a regular global section $W$ of $\mathcal{L}$, there is a triangulated functor

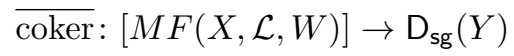

such that the composition

$$
[M F(X, \mathcal{L}, W)]_{\text {naive }} \rightarrow[M F(X, \mathcal{L}, W)] \stackrel{\overline{\text { coker }}}{\longrightarrow} \mathrm{D}_{\mathrm{sg}}(Y)
$$

is the functor coker.

Theorem 6.3. Let $X$ be a scheme that is projective over a Noetherian ring, $\mathcal{L}=$ $\mathcal{O}_{X}(1)$ the corresponding very ample line bundle, and $W$ a regular global section of $\mathcal{L}$. Define $Y \hookrightarrow X$ to be the zero subscheme of $W$. Then the triangulated functor

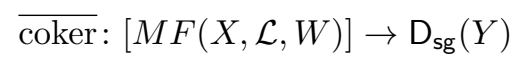

is fully faithful.

Proof. We will show there is a fully faithful functor

$$
\overline{\text { coker }}^{\prime}:[M F]_{\mathbb{H}} \rightarrow \mathrm{D}_{\mathrm{sg}}(Y)
$$

such that $\overline{\operatorname{coker}}^{\prime} \circ H=$ coker, where $H:[M F]_{\text {naive }} \rightarrow[M F]_{\mathbb{H}}$ is the functor defined in (지). To see this implies the Theorem, let $F:[M F]_{\text {naive }} \rightarrow[M F]$ be the canonical functor, and $G:[M F] \rightarrow[M F]_{\mathbb{H}}$ the equivalence of Theorem 12 ; note that $H=$ $G \circ F$. Consider the commutative diagram:

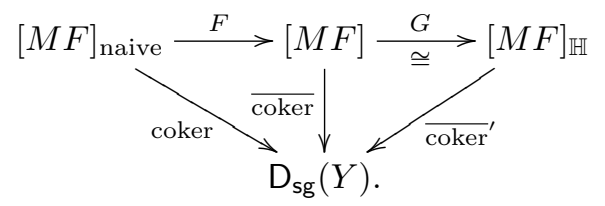

Since $\overline{\text { coker }}^{\prime} \circ G \circ F=$ coker $=\overline{\text { coker }} \circ F$, we have that $\overline{\text { coker }}^{\prime} \circ G \cong \overline{\text { coker }}$ by the universal property of Verdier quotients. Thus if $\overline{c o k e r}^{\prime}$ is fully faithful, so will be coker.

To define the functor $\overline{\operatorname{coker}}^{\prime}$, we set $\overline{\operatorname{coker}}^{\prime}(\mathbb{E})=$ coker $\mathbb{E}$. By Lemma a quasi-isomorphism

$$
\underline{\operatorname{Hom}}_{\mathrm{MF}}(\mathbb{E}, \mathbb{F}) \stackrel{\sim}{\rightarrow} i_{*} \underline{\operatorname{Hom}}_{\mathcal{O}_{Y}}\left(i^{*} \mathbb{E}, \operatorname{coker} \mathbb{F}\right)
$$

that is natural in both arguments, and thus there is a natural isomorphism

$$
\mathbb{H}^{0}\left(X, \underline{\operatorname{Hom}}_{\mathrm{MF}}(\mathbb{E}, \mathbb{F})\right) \cong \mathbb{H}^{0}\left(Y, \underline{\operatorname{Hom}}_{\mathcal{O}_{Y}}\left(i^{*} \mathbb{E}, \operatorname{coker} \mathbb{F}\right)\right) .
$$

By Theorem 5 there is an isomorphism

$$
\mathbb{H}^{0}\left(Y, \underline{\operatorname{Hom}}_{\mathcal{O}_{Y}}\left(i^{*} \mathbb{E}, \operatorname{coker} \mathbb{F}\right)\right) \cong \operatorname{Hom}_{\mathrm{D}_{\mathrm{sg}}(Y)}(\operatorname{coker} \mathbb{E}, \operatorname{coker} \mathbb{F})
$$


that is natural in both arguments. By composing these, we obtain a natural map

$$
\operatorname{Hom}_{[M F]_{\mathbb{H}}}(\mathbb{E}, \mathbb{F})=\mathbb{H}^{0}\left(X, \underline{\operatorname{Hom}}_{\mathrm{MF}}(\mathbb{E}, \mathbb{F})\right) \rightarrow \operatorname{Hom}_{\mathrm{D}_{\mathrm{sg}}(Y)}(\operatorname{coker} \mathbb{E}, \operatorname{coker} \mathbb{F})
$$

determining the functor $\overline{\text { coker }}^{\prime}$, which is a fully faithful since this map is bijective.

We have left to prove that $\overline{\operatorname{coker}}^{\prime} \circ H=$ coker. Both sides agree on objects, but we need to show they induce the same map on morphisms. Consider the diagram:

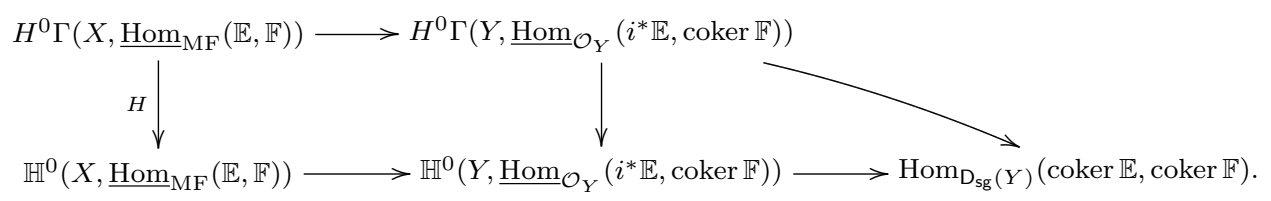

The right triangle is commutative by Theorem 5.7. The left hand square is commutative as the horizontal maps are induced by the map of chain complexes

$$
\underline{\operatorname{Hom}}_{\mathrm{MF}}(\mathbb{E}, \mathbb{F}) \rightarrow i_{*} \underline{\operatorname{Hom}}_{\mathcal{O}_{Y}}\left(i^{*} \mathbb{E}, \operatorname{coker} \mathbb{F}\right)
$$

and $H^{0}(-) \rightarrow \mathbb{H}^{0}(-)$ is a natural transformation. The composition of the bottom arrows in the diagram is by definition the map on morphisms induced by the functor $\overline{\text { coker }}^{\prime}$. Thus by the commutativity of the diagram, the map on morphisms induced by $\overline{\text { coker }}^{\prime} \circ H$ is equal to the composition of the top two arrows of the diagram. To finish the proof it is now enough to show that the following diagram commutes:

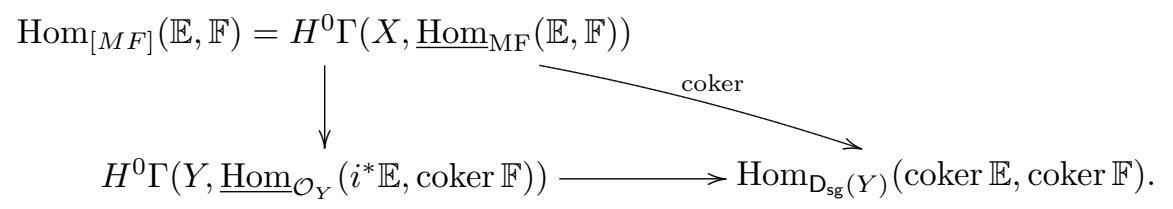

This follows from the definition of the map $\underline{\operatorname{Hom}}_{\mathrm{MF}}(\mathbb{E}, \mathbb{F}) \rightarrow i_{*} \underline{\operatorname{Hom}}_{\mathcal{O}_{Y}}\left(i^{*} \mathbb{E}\right.$, coker $\left.\mathbb{F}\right)$ given in (the proof of) $5 \cdot 2(3)$.

Remark 6.4. Theorem [6.3 has been proved in [0,3.14] in case $X$ is a smooth stack. Orlov proves an analogue in [四] without assuming $X$ is projective over an affine scheme. However he assumes that $\mathcal{L}=\mathcal{O}_{X}$. His Theorem does not seem to imply ours, nor does ours imply his, as $\mathcal{O}_{X}$ is not very ample in general. Lin and Pomerleano, in [3], give a different proof of Orlov's Theorem in case $X$ is defined over $\mathbb{C}$ and smooth. Finally Positselski, in [ [8], proves an analogue of Theorem $\mathbf{6 . 3}$ using exotic derived categories, which does not require $X$ to be projective. He does not require the coherent sheaves in the definition of matrix factorization to be locally free.

Theorem $[\mathbf{6 . 8}$ will show that the objects defined below are exactly the cokernels of matrix factorizations in $\mathrm{D}_{\mathrm{sg}}(Y)$, under the assumptions of Theorem $\mathbf{6}$. $]$.

Definition 6.5. Let $i$ : $Y \hookrightarrow X$ be a closed immersion of finite flat dimension. An object $\mathcal{F}$ in $D^{b}(Y)$ is relatively perfect on $Y$ if $i_{*} \mathcal{F}$ is perfect on $X$. We write $\operatorname{RPerf}(Y \hookrightarrow X)$ for the full subcategory of $D^{b}(Y)$ whose objects are relatively perfect on $X$. 
Since $i$ has finite flat dimension, $\operatorname{Perf}(Y)$ is a thick subcategory of $\operatorname{RPerf}(Y \hookrightarrow X)$. We define the relative singularity category of $i$ to be the Verdier quotient

$$
\mathrm{D}_{\mathrm{sg}}^{\mathrm{rel}}(Y \hookrightarrow X):=\frac{\operatorname{RPerf}(Y \hookrightarrow X)}{\operatorname{Perf}(Y)} .
$$

The canonical functor

$$
\mathrm{D}_{\mathrm{sg}}^{\mathrm{rel}}(Y \hookrightarrow X) \rightarrow \mathrm{D}_{\mathrm{sg}}(Y)
$$

is fully faithful and we may thus identify $\mathrm{D}_{\mathrm{sg}}^{\mathrm{rel}}(Y \hookrightarrow X)$ with a full subcategory of $\mathrm{D}_{\mathrm{sg}}(Y)$.

Remark 6.6. In this same context, a relative singularity category has also been defined by Positselski in [ $[\mathbf{8}]$. In general these two categories need not be equivalent, but we point out a relation between the two in 5 .

The following establishes one half of Theorem $\mathbf{6 . 8}$ (under milder assumptions):

Lemma 6.7. Assume $X$ is a Noetherian separated scheme of finite Krull dimension and that $X$ has enough locally frees (i.e., every coherent sheaf on $X$ is the quotient of a locally free coherent sheaf). Let $\mathcal{L}$ be a line bundle on $X$, assume $W$ a regular global section of $\mathcal{L}$ and let $i: Y \hookrightarrow X$ be the zero subscheme of $W$. For every object $\mathcal{G}$ of $\mathrm{D}_{\mathrm{sg}}^{\mathrm{rel}}(Y \hookrightarrow X)$, there exists a matrix factorization $\mathbb{E}$ and an isomorphism $\mathcal{G} \cong$ coker $\mathbb{E}$ in $\mathrm{D}_{\mathrm{sg}}^{\mathrm{rel}}(Y \hookrightarrow X)$.

Proof. Let $\mathcal{F}$ be a right bounded complex of locally free coherent sheaves on $Y$ that maps quasi-isomorphically to $\mathcal{G}$. Such a complex exists since $X$, and hence $Y$, has enough locally frees. Let $\mathcal{F} \leqslant k$ denote the brutal truncation of $\mathcal{F}$ in degree $k$. For any $k$, the cone of the canonical map $\mathcal{F} \rightarrow \mathcal{F} \leqslant k$ is a perfect complex and hence $\mathcal{F} \rightarrow \mathcal{F} \leqslant k$ is an isomorphism in $\mathrm{D}_{\text {sg }}^{\text {rel }}(Y \hookrightarrow X)$. Taking $k \ll 0$, the complex $\mathcal{F} \leqslant k$ is exact except in degree $k$, and hence we have reduced to the case where $\mathcal{G}=\mathcal{M}[-k]$ for some coherent sheaf $\mathcal{M}$ and integer $k$. Since coker is triangulated, we may assume $k=0$.

Since $i_{*} \mathcal{G}$ is a perfect complex, $i_{*} \mathcal{M}$ is locally of finite projective dimension. In fact, for all $x \in X$, the projective dimension of $i_{*} \mathcal{M}_{x}$ as a $\mathcal{O}_{X, x}$-module is at most $d:=\operatorname{dim}(X)$. Consider again a resolution $\mathcal{F}$ of $\mathcal{M}$ by locally free coherent sheaves on $Y$. Since a locally free coherent sheaf on $Y$ is locally of projective dimension one as a coherent sheaf on $X$, a high enough syzygy of this resolution of $\mathcal{M}$ will also be locally of projective dimension one on $X$. Specifically, the only non-zero cohomology sheaf of $i_{*} \mathcal{F} \leqslant-d$ will be locally of projective dimension one on $X$, and since $\mathcal{F} \leqslant-d \cong \mathcal{M}=\mathcal{G}$ in $\mathrm{D}_{\mathrm{sg}}^{\text {rel }}(Y \hookrightarrow X)$, we may assume $\mathcal{G}=\mathcal{M}$ where $\mathcal{M}$ is a coherent sheaf on $Y$ such that $i_{*} \mathcal{M}$ is locally of projective dimension one on $X$.

Now consider any surjection $\mathcal{E}_{0} \rightarrow i_{*} \mathcal{M}$ with $\mathcal{E}_{0}$ a locally free coherent sheaf on $X$. Since $i_{*} \mathcal{M}$ is locally of projective dimension one, the kernel $\mathcal{E}_{1}$ of this surjection is locally free. That is, we have a resolution of the form

$$
0 \longrightarrow \mathcal{E}_{1} \stackrel{\alpha}{\longrightarrow} \mathcal{E}_{0} \longrightarrow i_{*} \mathcal{M} \longrightarrow 0
$$


with $\mathcal{E}_{0}, \mathcal{E}_{1}$ locally free on $X$. In the diagram

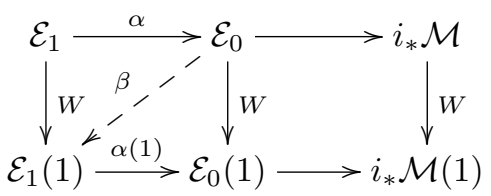

the right-most map is the zero map, and hence there exists diagonal arrow $\beta$ as shown causing both triangles to commute. This determines a matrix factorization $\mathbb{E}$ with $\operatorname{coker}(\mathbb{E}) \cong \mathcal{M}=\mathcal{G}$.

Theorem 6.8. Let $X$ be a scheme that is projective over a Noetherian ring of finite Krull dimension, $\mathcal{L}=\mathcal{O}_{X}(1)$ the corresponding very ample line bundle, and $W$ a regular global section of $\mathcal{L}$. Define $Y \hookrightarrow X$ to be the zero subscheme of $W$. The functor

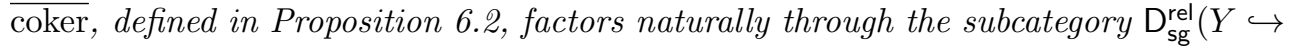
$X)$ of $\mathrm{D}_{\mathrm{sg}}(Y)$ and the induced map is an equivalence of triangulated categories:

$$
[M F(X, \mathcal{L}, W)] \stackrel{\cong}{\rightarrow} \mathrm{D}_{\mathrm{sg}}^{\text {rel }}(Y \hookrightarrow X) .
$$

In particular, if $X$ is regular, then we have an equivalence of triangulated categories

$$
\text { coker: }[M F(X, \mathcal{L}, W)] \stackrel{\cong}{\longrightarrow} \mathrm{D}_{\mathrm{sg}}(Y) .
$$

Proof. For any matrix factorization $\mathbb{E}=\left(\mathcal{E}_{1} \stackrel{e_{1}}{\longrightarrow} \mathcal{E}_{0} \stackrel{e_{0}}{\longrightarrow} \mathcal{E}_{1}\right)$ there is a short exact sequence of coherent sheaves on $X$

$$
0 \rightarrow \mathcal{E}_{1} \stackrel{e_{1}}{\rightarrow} \mathcal{E}_{0} \rightarrow i_{*} \operatorname{coker}(\mathbb{E}) \rightarrow 0 .
$$

Indeed, since $W$ is regular section, the composition $e_{0} \circ e_{1}=W: \mathcal{E}_{1} \rightarrow \mathcal{E}_{1}(1)$ is injective and hence $e_{1}$ must be. Thus $i_{*} \operatorname{coker}(\mathbb{E})$ is perfect and coker factors through the subcategory $\mathrm{D}_{\mathrm{sg}}^{\text {rel }}(Y \hookrightarrow X)$. On the other hand, for any object $\mathcal{F}$ in $\mathrm{D}_{\mathrm{sg}}^{\text {rel }}(Y \hookrightarrow X)$, there is a matrix factorization $\mathbb{E}$ such that coker $\mathbb{E} \cong \mathcal{F}$ by Lemma

The final assertion holds since every bounded complex of coherent sheaves on a regular scheme is perfect.

Remark 6.9. Let $i: Y \hookrightarrow X$ be as above. Let $\mathcal{T}$ be the thick subcategory of $\mathrm{D}_{\mathrm{sg}}(Y)$ generated by the objects $\mathbb{L} i^{*} \mathcal{M}$, where $\mathcal{M}$ ranges over all objects of $\mathrm{D}_{\mathrm{sg}}(X)$. Positselski defines in [8] the relative singularity category of $i: Y \hookrightarrow X$, which we denote ${ }^{\mathrm{P}} \mathrm{D}_{\text {sg }}^{\text {rel }}(Y \hookrightarrow X)$, to be the Verdier quotient $\mathrm{D}_{\mathrm{sg}}(Y) / \mathcal{T}$. It is easy to check that $\mathrm{D}_{\text {sg }}^{\text {rel }}(Y \hookrightarrow$ $X)$ is a subcategory of

$$
\mathcal{T}^{\perp}:=\left\{\mathcal{N} \in \mathrm{D}_{\mathrm{sg}}^{\text {rel }}(Y) \mid \operatorname{Hom}_{\mathrm{D}_{\mathrm{sg}}(Y)}(\mathcal{M}, \mathcal{N})=0 \text { for all objects } \mathcal{M} \in \mathcal{T}\right\} .
$$

This implies, keeping in mind the definition of morphisms in $\mathrm{D}_{\mathrm{sg}}(Y) / \mathcal{T}$, that the composition of the functors

$$
\mathrm{D}_{\mathrm{sg}}^{\mathrm{rel}}(Y \hookrightarrow X) \rightarrow \mathrm{D}_{\mathrm{sg}}(Y) \rightarrow \mathrm{D}_{\mathrm{sg}}(Y) / \mathcal{T}=:{ }^{\mathrm{p}} \mathrm{D}_{\mathrm{sg}}^{\mathrm{rel}}(Y \hookrightarrow X)
$$

is fully faithful.

This fully faithful functor need not be an equivalence. Indeed, let $k$ be a field and let $Q=k[[x, y]] /\left(x^{2}\right)$ and $R=Q /\left(y^{2}\right)=k[[x, y]] /\left(x^{2}, y^{2}\right)$. Let $Y:=\operatorname{Spec} R \hookrightarrow$ $\operatorname{Spec} Q=: X$ be the natural inclusion. If (四) were an equivalence, this would imply 
that the smallest thick subcategory of $\mathrm{D}_{\mathrm{sg}}(Y)$ containing $\mathrm{D}_{\mathrm{sg}}^{\text {rel }}(Y \hookrightarrow X)$ and $\mathcal{T}$ were all of $\mathrm{D}_{\mathrm{sg}}(Y)$. However we claim that the residue field $k$ is not in the thick subcategory generated by $\mathrm{D}_{\text {sg }}^{\text {rel }}(Y \hookrightarrow X)$ or $\mathcal{T}$. Indeed, it is easy to check that objects in either of these categories have periodic free resolutions, but the ranks of the free modules in a minimal free resolution of $k$ grow linearly and so $k$ cannot have a periodic resolution.

\section{References}

[1] Ragnar-Olaf Buchweitz. Maximal Cohen-Macaulay modules and Tatecohomology over Gorenstein rings. Unpublished manuscript, available at https://tspace.library.utoronto.ca/handle/1807/16682, 1987.

[2] David Eisenbud. Homological algebra on a complete intersection, with an application to group representations. Trans. Amer. Math. Soc., 260(1):35-64, 1980.

[3] Kevin H. Lin and Daniel Pomerleano. Global matrix factorizations. arXiv:1101.5847.

[4] Dmitri O. Orlov. Matrix factorizations for nonaffine LG-models. Math. Ann., 353(1):95-108, 2012.

[5] Dmitri O. Orlov. Triangulated categories of singularities and D-branes in Landau-Ginzburg models. Tr. Mat. Inst. Steklova, 246(Algebr. Geom. Metody, Svyazi i Prilozh.):240-262, 2004.

[6] Dmitri O. Orlov. Triangulated categories of singularities, and equivalences between Landau-Ginzburg models. Mat. Sb., 197(12):117-132, 2006.

[7] Alexander Polishchuk and Arkady Vaintrob. Matrix factorizations and singularity categories for stacks. arXiv:1011.4544.

[8] Leonid Positselski. Coherent analogues of matrix factorizations and relative singularity categories. arXiv:1102.0261.

[9] Daniel Quillen. Higher algebraic K-theory. I. In Algebraic K-theory, I: Higher K-theories (Proc. Conf., Battelle Memorial Inst., Seattle, Wash., 1972), pages 85-147. Lecture Notes in Math., Vol. 341. Springer, Berlin, 1973.

[10] Ed Segal. The closed state space of affine Landau-Ginzburg B-models. J. Noncommutative Geometry. To appear.

[11] Ian Shipman. A geometric approach to Orlov's theorem. Comp. Math. To appear.

Jesse Burke jburke@math.uni-bielefeld.de

Department of Mathematics, Universität Bielefeld, 33501 Bielefeld, Germany

Mark E. Walker mwalkerbounL.edv

Department of Mathematics, University of Nebraska, Lincoln, NE 68588 USA 